\title{
On the Interaction and Competition among Internet Service Providers
}

\author{
Sam C.M. Lee John C.S. Lui ${ }^{+}$
}

\begin{abstract}
The current Internet architecture comprises of different privately owned Internet service providers (ISPs) where higher tier ISPs supply connectivity service to lower tier ISPs and charge these ISPs for the transit service. For the higher tier ISPs, the main concern is how to increase the profit by attracting more lower tier ISPs (or traffic), while the lower tier ISPs concern about the connectivity, quality of service as well as the cost of the transit service. In this work, we seek to understand the interaction between different tiers of ISPs. Note that the lower tier ISPs can transmit traffic to each other, either by purchasing the service from higher tier ISPs, or by setting "private peering links" between themselves. Higher tier ISPs, on the other hand, cannot charge the transit service at will since there is competition among higher tier ISPs. We model the interaction of these ISPs via a game theoretic approach. We study the issues of (a) impact of private peering relationship among the lower tier ISPs, (b) under a competitive market, how can the higher tier ISPs perform resource allocation and revenue maximization so that resource monopoly can be avoided, and (c) conditions wherein higher tier ISPs are willing to perform network upgrade, in particular, when we scale up the network. Our mathematical framework provides insights on the interaction among ISPs and shows these ISPs can still gain profits as they upgrade the network infrastructures. Extensive simulations are carried out to quantify and support our theoretical claims.
\end{abstract}

Keywords: ISP interaction, competition, pricing, peering, resource allocation, network scaling

\section{Introduction}

The current Internet is formed by many different internet service providers (ISPs) which provide connectivity and transit service. One of the main problems facing ISPs is how to increase the profit and at the same time, sustain good performance as the Internet grows. One can loosely classify the ISPs into two types: (a) higher tier ISPs which cover large geographical areas and provide transit service, and (b) lower tier ISPs which provide connectivity service to regional users. For the lower tier ISPs, in order to gain the Internet access, they need to purchase the transit service from higher tier ISPs. These higher tier ISPs set their prices based on the service provisioning, and the prices depend on the allocated bandwidth, the amount of transferred traffic, as well as the competitive prices from other higher tier ISPs. Note that for lower tier ISPs which are within the same region, they have an option to set up "private peering links" among themselves so that they can bypass the higher tier ISPs and reduce their operating costs. The basic nature of the peering relationship is to exchange local traffic between the two lower tier ISPs via the peering link without paying the higher tier ISPs for the traffic transfer. In practice, these private

\footnotetext{
${ }^{+}$Sam C.M. Lee and John C.S. Lui are with the Department of Computer Science and Engineering, The Chinese University of Hong
} Kong. John C.S. Lui is the contact author, email: cslui@cse.cuhk.edu.hk. 
peering agreements can be quite complicated, involving many business considerations [7], [16], [4]. In [6], authors discuss how a provider should price its services differentially based on their characteristics such that prices can match service qualities. Authors in [5] also discuss how to present a cooperative pricing strategy to provide a fair distribution of profits to ISPs.

In [1], [10], [18], [21], authors study the economics of network pricing with multiple ISPs on the Internet. These authors all study a basic question of how to set prices for the Internet services so as to fairly share revenues among providers and at the same time, encourage the network to grow. However, these work underestimate the impact of two important factors: (a) pricing competition among higher tier ISPs and, (b) local peering relationship on the traffic demand. These factors have great impact on the proper pricing strategy. The aim of this work is to seek a fundamental understanding of the interaction between ISPs with peering links and competition. We explore how the peering relationship and competition among higher tier ISPs can affect the service purchasing strategies and pricing strategies.

For a lower tier ISP, it has two options to communicate with another lower tier ISP: either use the connection provided by higher tier ISPs, or to use the private peering link connecting between the two peers (if available). Even for the simple case of a constant bandwidth demand, deciding on the proper routing via these two connections is not a trivial task. Another factor which makes the decision of traffic allocation difficult is that all lower tier ISPs want to maximize their own utilities, and at the same time, reduce their operating costs (or payment) to the higher tier ISPs. Also, an ISP's strategy may depend on strategies taken by other ISPs, as well as the pricing policies employed by the higher tier ISPs. All these make it a challenging task to come up with an efficient transmission and routing strategy.

For the higher tier ISP, it needs to provide connectivity among lower tier ISPs. The main goal is to maximize its profit by attracting more potential customers (e.g., lower tier ISPs). To maximize the profit, a good pricing strategy is essential. In general, a higher tier ISP needs to address:

1. Profit Maximization : under a competitive market, does it exist a unique price by which the higher tier ISP's profit can be maximized under a homogeneous pricing scheme (i.e., all lower tier ISPs are charged using the same price)? If it exists, how can one determine this optimal price?

2. Bandwidth Allocation: how should the higher tier ISP allocate the bandwidth to competing lower tier ISPs and avoid the monopolization of bandwidth resource by a small subset of lower tier ISPs?

3. Network Scaling and Capacity Upgrade: when the number of lower tier ISPs increases, is there any incentive for the higher tier ISP to upgrade the network infrastructures such as the backbone capacity? Can the increase in revenue compensate for the increase in cost of deploying new services?

4. Impact of Private Peering: what is the impact of private peering relationships between the lower tier ISPs have on the higher tier ISP's pricing decision?

Take an example, some tier 1 ISPs, say AT \& T and Verizon Business are providing transit service 
to some tier 2 ISPs, PCCW and Entanet. AT \& T and Verizon Business are the higher tier ISPs, while PCCW and Entanet are the lower tier ISPs. Besides buying transit service from higher tier IPSs, PCCW and Entanet can establish a peering relationship under a service level agreement (SLA) between them. They can then send data to each other with a lower or even zero cost. The considering issues of buying transit service or not are the payment and the quality of the service.

The contribution of our paper is to answer the above questions. We use a "game theoretic" approach to study the interaction of the two types of ISPs and illustrate the impact of private peering in the competitive market. In particular,

1. We present a generalized competitive model that captures the pricing competition among the higher tier ISPs and traffic demand and routing decision of the lower tier ISPs. This two-tiers-interaction represents a basic framework of the current Internet.

2. We show how lower tier ISPs can distributively determine their transmission and routing decisions via a convex optimization method.

3. We propose a distributed algorithm for higher tier ISPs to allocate their bandwidth resources to lower tier peers so as to avoid resource monopolization.

4. We show how a higher tier ISP can infer an optimal pricing so as to maximize its profit even under a competitive environment among other higher tier ISPs.

5. Last but not least, we consider the issue of network scaling (when we increase the number of ISPs) and derive conditions wherein higher tier ISPs have the incentive to upgrade the backbone capacity.

\section{Network and Game-theoretic Models}

In this section, we describe our network model and formulate mathematical models for various ISPs. Figure 1 depicts our network model. The network consists of $n$ lower tier ISPs and $m$ higher tier ISPs. Lower tier ISPs can communicate with each other by sending traffic via the private peering links between themselves, or through the links connecting to the higher tier ISPs. To provide connection service for various lower tier ISPs, each higher tier ISP $k \in\{1,2, \ldots, m\}$, has a communication network (in which we abstract it as a link) that has a total capacity of $n_{k} \mathcal{C}^{k}$ (in units of bps). For each lower tier ISP $i \in\{1,2, \ldots, n\}$, it possesses at most $m$ links to each higher tier ISPs and possibly $n-1$ private peering links to other lower tier peers. The private peering link between peer $i$ and peer $j$ is denoted as $l_{i j}$ and this link has a capacity of $c_{i j}$ (in unit of bps). Note that if we set $c_{i j}=0$, it implies that there is no peering link between peer $i$ and peer $j$. The link connecting lower tier ISP $i$ and the higher tier ISP $k$ is denoted as $l_{i}^{k}$, and the higher tier ISP $k$ allocates $\mathcal{C}_{i}^{k}$ amount of bandwidth (in units of bps) for this connection. Note that if we set $\mathcal{C}_{i}^{k}=0$, it implies that the lower tier ISP $i$ is not connected to ISP $k$. Let $n_{k}$ be the number of lower tier ISPs buying connection service from the higher tier ISP $k$. We also denote $\mathcal{G}_{i}$ as the set of higher tier ISPs in which the lower tier ISP $i$ is buying connection service from, and $\mathcal{H}_{k}$ as the 
set of lower tier ISPs in which the higher tier ISP $k$ is providing connection service to. Note that this network model is a generalization of the network model in [12] wherein only a single higher tier ISP was considered (therefore, in [12], there is no competition among higher tier ISPs). Table I lists all notations used in our network model.

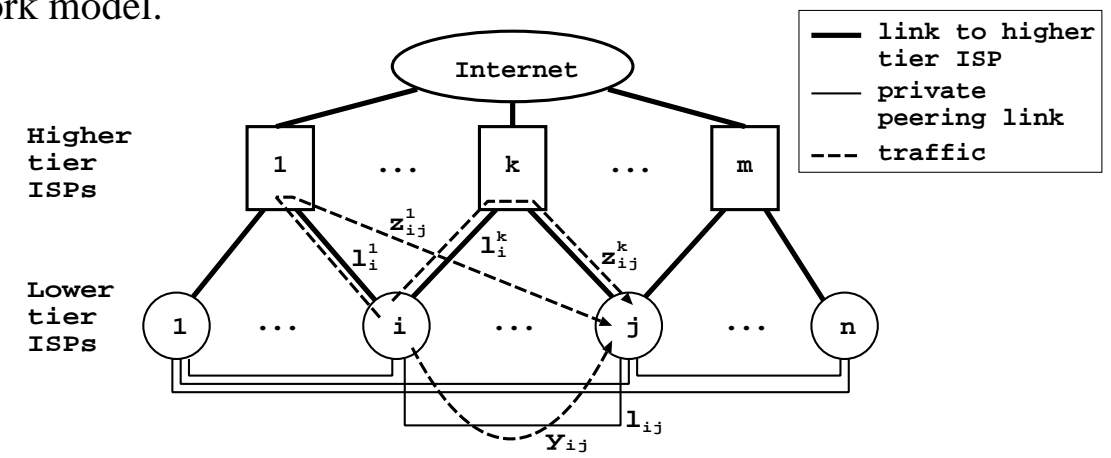

Fig. 1. A network representing $n$ lower tier ISPs and $m$ higher tier ISPs. Each lower tier ISP has at most $m$ links to the high tier ISPs and possibly $n-1$ private peering links with other lower tier ISPs. The lower tier ISP $i$ can communicate with lower tier ISP $j$ in two ways: via the peering link $l_{i j}$ or via the higher tier ISP links $l_{i}^{k}$ s. The traffic rate on link $l_{i j}$ is $y_{i j}$ while the traffic rate on link $l_{i}^{k}$ is $z_{i j}^{k}$.

Let $x_{i j}$ denote the traffic transmission rate (in unit of bps) from lower tier ISP $i$ to lower tier ISP $j$. If the transmission rate can be supported, then the lower tier ISP $i$ obtains a utility of $A_{i j}\left(x_{i j}\right)$ where $A_{i j}$ is a strictly concave function in $x_{i j}$. As indicated in [9], concave function is commonly used to represent elastic traffic, which is the dominant traffic in the Internet. In here, a weighted log function is used such that $A_{i j}\left(x_{i j}\right)=w_{i j} \log \left(1+x_{i j}\right)$. The weighting $w_{i j}$ can be interpreted as the "happiness weighting coefficient" of transmitting traffic from the lower tier ISP $i$ to the lower tier ISP $j$. If $w_{i j}>w_{i s}$, it implies that ISP $i$ prefers to communicate with ISP $j$ than with ISP $s$. Note that the log function is chosen as it leads to a proportionally fair resource allocation if proper congestion control is used. Additionally, this type of utility function is also used for performing distributed admission control [1].

For the traffic from lower tier ISP $i$ to $j$ it can either go through the private link $l_{i j}$, or through any mutually connected higher tier ISPs. We denote $y_{i j}$ as the traffic rate that ISP $i$ decides to transmit through the link $l_{i j}$, and $z_{i j}^{k}$ as the traffic rate through the link of ISP $k, l_{i}^{k}$. Therefore, the traffic rate $x_{i j}$ is equal to

$$
x_{i j}=y_{i j}+\sum_{k \in \mathcal{G}_{i}} z_{i j}^{k} \quad \text { for } i, j \in\{1, \ldots, n\} .
$$

One special case is for the traffic rate $x_{i i}$, representing the traffic from ISP $i$ to destinations other than the $n-1$ lower tier ISPs, which are outside this network. This type of traffic has to go through the higher tier ISPs, we have

$$
y_{i i}=0 \quad \text { and } \quad x_{i i}=\sum_{k \in \mathcal{G}_{i}} z_{i i}^{k} \quad \text { for } i \in\{1, \ldots, n\} .
$$


$m: \quad$ Number of higher tier ISPs in the communication network.

$n: \quad$ Number of lower tier ISPs in the communication network.

$n_{k}: \quad$ Number of lower tier ISPs buying connection service from the higher tier ISP $k$.

$l_{i}^{k}: \quad \quad \quad$ An abstraction of the communication link between lower tier ISP $i$ and the higher tier ISP $k$.

$l_{i j}: \quad$ The private peering link connecting lower tier ISP $i$ to lower tier ISP $j$.

$n_{k} \mathcal{C}^{k}: \quad$ Total capacity of the link of the higher tier ISP $k$.

$\mathcal{C}_{i}^{k}$ : $\quad$ Allocation of the higher tier ISP $k$ 's link bandwidth to the lower tier ISP $i$, i.e. capacity of link $l_{i}^{k}$.

$c_{i j}$ : $\quad$ Capacity of the private peering link $l_{i j}$ connecting ISP $i$ to ISP $j$.

$w_{i j}$ : The happiness weighting coefficient of transmitting traffic from ISP $i$ to ISP $j$.

$x_{i j}: \quad \quad \quad \quad \quad \quad \quad \quad \quad$ raffic transmission rate from the lower tier ISP $i$ to the lower tier ISP $j$, such that $x_{i j}=y_{i j}+\sum_{k \in \mathcal{G}_{i}} z_{i j}^{k}$.

$y_{i j}$ : $\quad$ Traffic transmission rate from the lower tier ISP $i$ to the lower tier ISP $j$ going through the private link $l_{i j}$.

$z_{i j}^{k}$ : $\quad$ Traffic transmission rate from the lower tier ISP $i$ to the lower tier ISP $j$ going through the higher tier ISP $k$ 's link $l_{i}^{k}$.

$z_{i}^{k}: \quad \quad \quad$ Aggregate traffic rate that the lower tier ISP $i$ sends through the higher tier ISP $k$ 's link.

$\bar{z}^{k}: \quad \quad \quad$ Aggregate traffic rate through the higher tier ISP $k$ 's link from all lower tier ISPs.

$\gamma: \quad$ The variable to map the congestion cost of the lower tier ISP into monetary value.

$\mathcal{P}_{i}^{k}: \quad \quad \quad \quad$ Price per unit bandwidth of the link of higher tier ISP $k$ for the lower tier ISP $i$. We assume $\mathcal{P}_{i}^{k}=\mathcal{P}^{k}$ for all $i$.

$p_{i j}$ : $\quad$ Price per unit bandwidth of the private peering link $l_{i j}$.

$\vec{y}_{i}: \quad \vec{y}_{i}=\left(y_{i 1}, y_{i 2}, \ldots, y_{i n}\right)$ denotes the traffic rate vector of lower tier ISP $i$ through its private links.

$\vec{z}_{i}^{k}: \quad \vec{z}_{i}^{k}=\left(z_{i 1}^{k}, z_{i 2}^{k}, \ldots, z_{i n}^{k}\right)$ denotes the traffic rate vector of lower tier ISP $i$ through the link of the higher tier ISP $k$.

$\mathcal{G}_{i}: \quad$ The set of higher tier ISPs providing connection service for lower tier ISP $i$.

$\mathcal{H}_{k}: \quad$ The set of lower tier ISPs buying connection service from the higher tier ISP $k$, i.e. $k \in \mathcal{G}_{i} \Leftrightarrow i \in \mathcal{H}_{k}$.

$\mathcal{S}_{i}: \quad$ The set of lower tier ISPs having peering links to lower tier ISP $i$.

$\mathcal{T}_{i}: \quad \quad \quad \quad$ The set of lower tier ISPs such that lower tier ISP $i$ wants to send traffic to, i.e. $w_{i j}>0$ if $j \in \mathcal{T}_{i}$.

\section{TABLE I}

NOTATIONS USED TO REPRESENT THE NETWORK BETWEEN $n$ LOWER TIER ISPS AND $m$ HIGHER TIER ISPS

To simplify notation, let $z_{i}^{k}=\sum_{j \in \mathcal{T}_{i}} z_{i j}^{k}$ be the aggregate traffic rate that ISP $i$ sends through the higher tier ISP $k$ and $\bar{z}^{k}=\sum_{i \in \mathcal{H}_{k}} z_{i}^{k}$ is the aggregate traffic from all lower tier ISPs to the higher tier ISP $k$, where $\mathcal{T}_{i}$ denotes the set of lower tier ISPs in which ISP $i$ wants to send data to (i.e. $w_{i j}>0$ if $j \in \mathcal{T}_{i}$ ).

For lower tier ISPs, if they want to transmit traffic to other lower tier ISPs, they need to pay the connected higher tier ISPs for the transit service. The price per unit bandwidth for ISP $i$ sending traffic along the link $l_{i}^{k}$ of the higher tier ISP $k$ is $\mathcal{P}_{i}^{k}$, and the price is determined by the higher tier ISP $k$. The lower tier ISP $i$ may also transmit the traffic through private link $l_{i j}$ (if it exists) and we let $p_{i j}$ be the price per unit bandwidth, and this price is mutually agreed upon between the two lower tier ISPs. Let $\vec{y}_{i}=\left(y_{i 1}, y_{i 2}, \ldots, y_{i n}\right)$ denote the traffic rate vector of the lower tier ISP $i$ that transmitting through its private links and $\vec{z}_{i}^{k}=\left(z_{i 1}^{k}, z_{i 2}^{k}, \ldots, z_{i n}^{k}\right)$ denote the traffic rate vector transmitting through the higher tier ISP $k$. Also, the higher tier ISP $k$ sets the following price vector $\overrightarrow{\mathcal{P}}^{k}=\left(\mathcal{P}_{1}^{k}, \mathcal{P}_{2}^{k}, \ldots, \mathcal{P}_{n}^{k}\right)$ for various lower tier ISPs.

Lower tier ISPs also care about the "quality" of their service. In here, we use congestion as an indicator for the quality of service. Assuming links can be represented as an M/M/1 queue [1], the delay on the 
link can be treated as a congestion indication. In order to provide these congestion indicators to the connected lower tier ISPs, the higher tier ISP $k$ will perform appropriate bandwidth resource allocation (which will be presented in later section) and broadcast its allocation to the lower tier ISP $i$ as $\mathcal{C}_{i}^{k}$. Under this framework, the congestion cost of link $l_{i j}$ is $\frac{1}{c_{i j}-y_{i j}}$ and the cost of link $l_{i}^{k}$ is $\frac{1}{\mathcal{C}_{i}^{k}-z_{i}^{k}}$.

Now we formulate the mathematical optimization for each lower tier ISP. Considering the lower tier ISP $i$, it wants to maximize the following objective function:

$$
\begin{aligned}
\operatorname{Max} U_{i}= & \sum_{j \in \mathcal{T}_{i}} w_{i j} \log \left(1+y_{i j}+\sum_{k \in \mathcal{G}_{i}} z_{i j}^{k}\right)-\sum_{k \in \mathcal{G}_{i}} \mathbf{1}_{\left\{z_{i}^{k} \neq 0\right\}}\left[\frac{\gamma}{\mathcal{C}_{i}^{k}-z_{i}^{k}}\right]-\sum_{k \in \mathcal{G}_{i}} \mathcal{P}_{i}^{k} z_{i}^{k} \\
& -\sum_{j \in \mathcal{S}_{i}} \mathbf{1}_{\left\{y_{i j} \neq 0\right\}}\left[\frac{\gamma}{c_{i j}-y_{i j}}\right]-\sum_{j \in \mathcal{S}_{i}} p_{i j} y_{i j} \\
\text { s. t. } \quad & 0 \leq y_{i j} \leq c_{i j} \text { for all } j \in \mathcal{S}_{i}, \quad y_{i j}=0 \text { for all } j \in \mathcal{T}_{i}-\mathcal{S}_{i}, \\
& \sum_{j \in \mathcal{T}_{i}} z_{i j}^{k} \leq \mathcal{C}_{i}^{k} \text { for all } k \in \mathcal{G}_{i}, \quad z_{i j}^{k} \geq 0 \text { for all } j \in \mathcal{T}_{i} \text { and } k \in \mathcal{G}_{i} .
\end{aligned}
$$

where $\mathbf{1}_{\{p\}}$ is an indicator function and the set $\mathcal{S}_{i}$ is the collection of peers having private peering links established with lower tier ISP $i$. The above objective function in Eq.(3) represents the economic incentive for ISP $i$ to carry out traffic transmission. The first term in the objective function $w_{i j} \log \left(1+y_{i j}+\sum_{k \in \mathcal{G}_{i}} z_{i j}^{k}\right)$ is the happiness of ISP $i$ in sending data to ISP $j$ through its connecting links. The second term $\frac{\gamma}{\mathcal{C}_{i}^{k}-z_{i}^{k}}$ is the congestion cost of ISP $i$ experienced in sending traffic through the link of the higher tier ISP $k$. The variable $\gamma>0$ represents the congestion cost impact to a lower tier ISP and it translates the congestion cost into an appropriate monetary value. Note that the larger the value of $\gamma$ indicates that lower tier ISPs are more concern about the congestion. In later section, we will show the the impact of $\gamma$ on the convergence point of the traffic transmission rates. Note that the congestion cost will be zero if ISP $i$ does not transmit via this link, or the ISP did not purchase transit service from the higher tier ISP $k$. The term $\mathcal{P}_{i}^{k} z_{i}^{k}$ is the total payment of lower tier ISP $i$ to the higher tier ISP $k$. Also, $\frac{\gamma}{c_{i j}-y_{i j}}$ is the congestion cost on the private peering link between lower tier ISPs $i$ and $j$ when the traffic rate on $l_{i j}$ is non-zero. Lastly, ISP $i$ needs to pay $p_{i j} y_{i j}$ to lower tier ISP $j$ for using the private link (note: it is possible to model free peering by setting $p_{i j}=p_{j i}=0$ ). Last but not least, the happiness, congestion cost and payment are mapped to the same monetary domain with $w_{i j}$ and $\gamma$. The constraints represented in Eq.(4) specify the feasible region of the optimization problem. The first constraints are non-negative and capacity constraints of the peering links. The second constraint is due to the absence of peering links. The third and fourth are the capacity and non-negative constraints of links of higher tier ISPs respectively. In summary, each lower tier ISP $i$ needs to determine the traffic rates vectors $\vec{y}_{i}$ and $\vec{z}_{i}^{*}$ for all $k \in \mathcal{G}_{i}$ so as to maximize its utility as defined in Eq.(3). 
It is important for us to point out that the optimization of different lower tier ISPs are dependent on each other. For each lower tier ISP $i$, given the bandwidth allocation $\mathcal{C}_{i}^{k}$ of the link of the higher tier ISP $k$, it performs an optimization and determines its transmission rate $z_{i}^{k}$ and bids for this allocation to ISP $k$. After collecting the bidding information from all connected lower tier ISPs, the higher tier ISP $k$ needs to determine the new bandwidth allocation based on these biddings and to perform the proper resource allocation (this will be discussed in Section IV).

In this paper, we model this form of interaction between ISPs as a "non-cooperative game". Under the game theoretic framework, for a given collection of price vectors of ISPs $\overrightarrow{\mathcal{P}}=\left(\mathcal{P}^{1}, \mathcal{P}^{2}, \ldots, \mathcal{P}^{m}\right)$, this defines a non-cooperative game between these $n$ lower tier ISPs [19]. They interact with each other and determine their optimal traffic rates. Given the existence of an equilibrium point, the operating point for $n$ lower tier ISPs is the solution to the Nash equilibrium of this game. For each collection of price vectors $\overrightarrow{\mathcal{P}}>0$, a Nash equilibrium point for this $n$-ISPs game is defined as two $n$-tuples $y^{*}=\left(\vec{y}_{1}^{*}, \vec{y}_{2}^{*}, \ldots, \vec{y}_{n}^{*}\right)$ and $z^{*}=\left(\vec{z}_{1}^{*}, \vec{z}_{2}^{*}, \ldots, \vec{z}_{n}^{*}\right)$, where $\vec{z}_{i}=\left(\vec{z}_{i}^{1}, \vec{z}_{i}^{2}, \ldots, \vec{z}_{i}^{m}\right)$, such that for all lower tier ISPs $i \in\{1,2, \ldots, n\}$ :

$$
U_{i}\left(y^{*}, z^{*}, \overrightarrow{\mathcal{P}}\right) \geq U_{i}(y, z, \overrightarrow{\mathcal{P}})
$$

for any other feasible traffic vector $y=\left(\vec{y}_{1}, \vec{y}_{2}, \ldots, \vec{y}_{n}\right)$ and $z=\left(\vec{z}_{1}, \vec{z}_{2}, \ldots, \vec{z}_{n}\right)$ that satisfies the constraints defined in Eq.(4). For a higher tier ISP, say $k$, it has to solve a profit maximization problem:

$$
\text { Maximize } \mathcal{P}^{k} \cdot \bar{z}^{k *}\left(\mathcal{P}^{k}\right) \quad \text { over } \mathcal{P} \geq 0
$$

where $\bar{z}^{k *}\left(\mathcal{P}^{k}\right)$ is the aggregate traffic on the link of ISP $k$ at the Nash equilibrium. In this work, we assume homogeneous pricing. Therefore, $\mathcal{P}_{i}^{k}=\mathcal{P}^{k}$ for all $i \in\{1, \cdots, n\}$ and $k \in\{1, \cdots, m\}$. This equivalently defines a Stackelberg game ${ }^{1}$ [19] with $m$ leaders (the higher tier ISPs) and $n$ non-cooperative Nash followers (the lower tier ISPs).

\section{Solution to the Utility Maximization Problem of Lower Tier ISPs}

In this section, we show how a lower tier ISP, say $i$, can determine its optimal transmission rates and routing. The transmission rate vector is $\vec{z}_{i}^{k}$ for $k \in \mathcal{G}_{i}$, to other lower tier ISPs via the links of higher tier ISPs, and $\vec{y}_{i}$, is the transmission rate vector via peering links. Assuming that the lower tier ISP knows all the prices $\mathcal{P}^{k}$ and the associated bandwidth allocations $\mathcal{C}_{i}^{k}$ for $k \in \mathcal{G}_{i}$ specified by the connected higher tier ISPs, one can model this as a convex optimization problem as defined in Eq.(3). In this section, we investigate the necessary and boundary conditions for a lower tier ISP to maximize its utility.

Necessary conditions for positive transmission rate: We first consider the case where the traffic rate is non-zero. Since $U_{i}$ is discontinuous at $y_{i j}=0$ (i.e., the traffic rate through the peering link $l_{i j}$ is

\footnotetext{
${ }^{1}$ A Stackelberg game is a strategic game in which there is a leader who makes the decision first, and then other players, knowing the decision of the leader, will then their decisions. So the leader has a first move advantage over the followers.
} 
zero) and $z_{i}^{k}=0$ (i.e., the traffic rate through the link of the higher tier ISP $k$ is zero), we first show the necessary conditions when $y_{i j} \neq 0$ and $z_{i}^{k} \neq 0$. The optimization of Eq.(3) has $\left|\mathcal{T}_{i}\right|\left|\mathcal{G}_{i}\right|+\left|\mathcal{S}_{i}\right|$ variables. The second order partial derivatives with respect to $y_{i j}$ and $z_{i j}^{k}$ are:

$$
\begin{aligned}
\frac{\partial^{2} U_{i}}{\partial y_{i j}^{2}} & =\frac{-w_{i j}}{\left(1+y_{i j}+\sum_{k \in \mathcal{G}_{i}} z_{i j}^{k}\right)^{2}}-\frac{2 \gamma}{\left(c_{i j}-y_{i j}\right)^{3}}<0, \\
\frac{\partial}{\partial z_{i j}^{k}} \frac{\partial U_{i}}{\partial z_{i j}^{k}} & =\frac{-w_{i j}}{\left(1+y_{i j}+\sum_{k \in \mathcal{G}_{i}} z_{i j}^{k}\right)^{2}}-\frac{2 \gamma}{\left(\mathcal{C}_{i}^{k}-z_{i}^{k}\right)^{3}}<0 .
\end{aligned}
$$

For $j_{1} \neq j_{2} \neq i \in \mathcal{S}_{i}$ and $k_{1} \in \mathcal{G}_{i}$, the second order partial derivatives of Eq.(3) with respect to $y_{i j}$ and $z_{i j}^{k_{1}}$ are:

$$
\frac{\partial^{2} U_{i}}{\partial y_{i j_{1}} \partial y_{i j_{2}}}=0, \quad \frac{\partial^{2} U_{i}}{\partial y_{i j_{1}} \partial z_{i j_{2}}^{k_{1}}}=0, \quad \frac{\partial^{2} U_{i}}{\partial y_{i j_{1}} \partial z_{i j_{1}}^{k_{1}}}=\frac{-w_{i j_{1}}}{\left(1+y_{i j_{1}}+\sum_{k \in \mathcal{G}_{i}} z_{i j_{1}}^{k}\right)^{2}}<0
$$

and for $j_{1} \neq j_{2} \neq i \in \mathcal{T}_{i}$ and $k_{1} \neq k_{2} \in \mathcal{G}_{i}$, the second order partial derivatives of Eq.(3) with respect to $y_{i j}$ and $z_{i j}^{k}$ are:

$$
\frac{\partial^{2} U_{i}}{\partial z_{i j_{1}}^{k_{1}} \partial z_{i j_{2}}^{k_{2}}}=0 ; \quad \frac{\partial^{2} U_{i}}{\partial z_{i j_{1}}^{k_{1}} \partial z_{i j_{2}}^{k_{1}}}=\frac{-2 \gamma}{\left(\mathcal{C}_{i}^{k_{1}}-z_{i}^{k_{1}}\right)^{3}}<0 ; \quad \frac{\partial^{2} U_{i}}{\partial z_{i j_{1}}^{k_{1}} \partial z_{i j_{1}}^{k_{2}}}=\frac{-w_{i j_{1}}}{\left(1+y_{i j_{1}}+\sum_{k \in \mathcal{G}_{i}} z_{i j_{1}}^{k}\right)^{2}}<0
$$

Therefore, the Hessian matrix of the objective function in Eq.(3) is negative definite on the non-negative space bounded by $y_{i j} \leq c_{i j}$ and $z_{i}^{k} \leq \mathcal{C}_{i}^{k}$. So $U_{i}$ is strictly concave in $y_{i j}$ for $j \in \mathcal{S}_{i}$ and $z_{i j}^{k}$ for all $j \in \mathcal{T}_{i}$ and $k \in \mathcal{G}_{i}$. The maximum utility and optimizer to this problem is unique and can be found by the Lagrangian method [20]. The necessary conditions of $y_{i j}$ for $j \in \mathcal{S}_{i}$ and $z_{i j}^{k}$ for $j \in \mathcal{T}_{i}$ and $k \in \mathcal{G}_{i}$ of the maximization of $U_{i}$ are:

$$
\frac{\partial U_{i}}{\partial y_{i j}}\left\{\begin{array}{ll}
<0 & \text { if } y_{i j}=0 \\
=0 & \text { if } y_{i j}>0
\end{array}, \quad \frac{\partial U_{i}}{\partial z_{i j}^{k}} \begin{cases}<0 & \text { if } z_{i j}^{k}=0 \\
=0 & \text { if } z_{i j}^{k}>0\end{cases}\right.
$$

Boundary conditions for positive transmission rate: Since the objective function Eq.(3) is discontinuous at the boundaries, the necessary conditions given above may not achieve the global maximum. We are now going to explore the boundary cases when the traffic rate tends to zero, i.e., $y_{i j}=0$ or $z_{i}^{k}=0$.

Figure 2 illustrates an example when the optimal traffic rate is at boundary. The utility of the lower tier ISP $i$ is plotted against one particular traffic rate $y_{i j}$ (against $z_{i j}^{k}$ is similar). Figure 2(a) shows the case when the optimal value of $\left.\frac{\partial U_{i}}{\partial y_{i j}}\right|_{y_{j}^{*}}=0<0$. Referring to the figure, the optimal value $y_{i j}^{o p t}=0$. Since there is no congestion cost in the peering link when $y_{i j}^{o p t}=0$, the maximum utility is at point $P_{1}$ rather than point $P_{2}$. 
Figure 2(b) shows the case when $y_{i j}^{*}=\arg \left\{\frac{\partial U_{i}}{\partial y_{i j}}=0\right\}$ is positive and the corresponding utility is point $P_{3}$. If the utility when $y_{i j}=0$ is at point $P_{2}$, which is smaller than the utility at $P_{3}$, then $P_{3}$ is the maximum point and $y_{i j}^{o p t}=y_{i j}^{*}$ is the corresponding traffic rate. However, there exists a possibility that the utility when $y_{i j}=0$ is at point $P_{1}$, which is larger than the utility at $P_{3}$. In this case, $P_{1}$ is the maximum point and $y_{i j}^{o p t}=0$.

Let us provide the physical interpretation of the two cases illustrated in Figure 2(b). If the utility at the boundary point is $P_{2}$, it indicates that when the traffic rate $y_{i j}$ increases from 0 to $y_{i j}^{*}$, the increase in happiness outweighs the aggregate increases in congestion cost and the payment, thus achieving the maximum utility at point $P_{3}$. But if the utility at the boundary point is $P_{1}$, it means that when the traffic rate increases, the increase in happiness cannot compensate for the increases in congestion cost and payment. So the best strategy for lower tier ISP $i$ is not to send data through the link $l_{i j}$. Note that when lower tier ISP $i$ does not send data through any link, it has zero utility. Therefore, a lower tier ISP can always achieve a non-negative utility, since in the worse case, it can opt not to transmit.

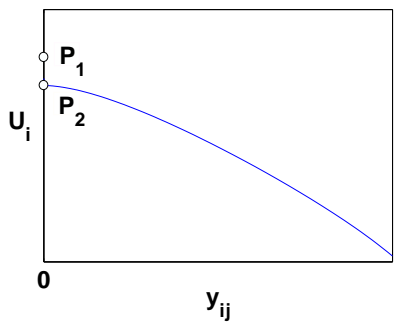

(a)

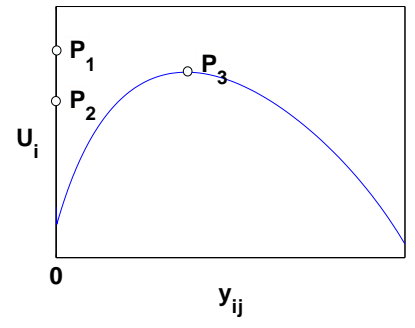

(b)

Fig. 2. Utility of lower tier ISP $i$ against one particular traffic rate $y_{i j}$, (a) when negative $y_{i j}^{*}$ (b) when positive $y_{i j}^{*}$.

Solution for lower tier ISPs: We end this section with an example showing how a lower tier ISP computes its optimal routing. Consider a network with three lower tier ISPs and two higher tier ISPs. Lower tier ISP 1 has a private link connecting to lower tier ISP 2 with a capacity $c_{12}=5$ and unit price $p_{12}=1$, and has no peering link connecting to lower tier ISP 3 . The happiness weighting coefficients for the lower tier ISP 1 are $w_{11}=5, w_{12}=10$ and $w_{13}=3$ with $\gamma=1$. The higher tier ISP 1 allocates a capacity $\mathcal{C}_{1}^{1}=20$ to lower tier ISP 1 with unit price $\mathcal{P}^{1}=1.1$ and the higher tier ISP 2 allocates a capacity $\mathcal{C}_{1}^{2}=30$ to lower tier ISP 1 with unit price $\mathcal{P}^{2}=1.2$. To determine the optimal transmission rates vectors of the lower tier ISP 1 , the necessary conditions with positive traffic rates are:

$$
\begin{array}{r}
\frac{10}{1+y_{12}+z_{12}^{1}+z_{12}^{2}}-\frac{1}{\left(5-y_{12}\right)^{2}}-1 \begin{cases}<0 & \text { if } y_{12}=0 \\
=0 & \text { if } y_{12}>0\end{cases} \\
\frac{10}{1+y_{12}+z_{12}^{1}+z_{12}^{2}}-\frac{1}{\left(20-z^{1}\right)^{2}}-1.1 \begin{cases}<0 & \text { if } z_{12}^{1}=0 \\
=0 & \text { if } z_{12}^{1}>0\end{cases}
\end{array}
$$




$$
\begin{aligned}
& \frac{10}{1+y_{12}+z_{12}^{1}+z_{12}^{2}}-\frac{1}{\left(30-z^{2}\right)^{2}}-1.2 \begin{cases}<0 & \text { if } z_{12}^{2}=0 \\
=0 & \text { if } z_{12}^{2}>0\end{cases} \\
& \frac{5}{1+z_{11}^{1}+z_{11}^{2}}-\frac{1}{\left(20-z^{1}\right)^{2}}-1.1 \begin{cases}<0 & \text { if } z_{11}^{1}=0 \\
=0 & \text { if } z_{11}^{1}>0\end{cases} \\
& \frac{5}{1+z_{11}^{1}+z_{11}^{2}}-\frac{1}{\left(30-z^{2}\right)^{2}}-1.2 \begin{cases}<0 & \text { if } z_{11}^{2}=0 \\
=0 & \text { if } z_{11}^{2}>0\end{cases} \\
& \frac{3}{1+z_{13}^{1}+z_{13}^{2}}-\frac{1}{\left(20-z^{1}\right)^{2}}-1.1 \begin{cases}<0 & \text { if } z_{13}^{1}=0 \\
=0 & \text { if } z_{13}^{1}>0\end{cases} \\
& \frac{3}{1+z_{13}^{1}+z_{13}^{2}}-\frac{1}{\left(30-z^{2}\right)^{2}}-1.2 \begin{cases}<0 & \text { if } z_{13}^{2}=0 \\
=0 & \text { if } z_{13}^{2}>0\end{cases}
\end{aligned}
$$

The solution to the above system of equations gives the optimal rate vectors of $y_{12}=2.39, \vec{z}^{1}=$ $\{3.56,5.48,1.66\}, \vec{z}^{2}=\{0,0,0\}$ and utility $U_{1}=17.70$.

\section{Distributed Bandwidth Allocation by Higher Tier ISPs}

For a higher tier ISP, it has to determine the proper bandwidth allocation to its connected customers (e.g., lower tier ISPs) and at the same time, it needs to make sure that there is no bandwidth monopolization by a small number of lower tier ISPs. Since a monopoly by a small number of ISPs surely reduces the customer size and customer diversity, which in turn increase the risk of running the service provisioning business. Moreover, in order to maximize its revenue or profit, a higher tier ISP has to know the demand in its link bandwidth. Given the total amount of link bandwidth resource $n_{k} \mathcal{C}^{k}$, higher tier ISP $k$ needs to determine how to distribute this common resource to the $n_{k}$ lower tier ISPs. In this work, we propose a distributed approach which we called the Equal Sharing Algorithm.

First, let us present the general framework under which the higher tier ISPs can interact with their customers so that they can discover the actual resource demands from these lower tier ISPs. Also, how lower tier ISPs are informed the pricing information and the bandwidth resources. Initially, each higher tier ISP $k$ for $k \in\{1,2, \ldots, m\}$ equally distributes its link capacity to all its connected lower tier ISP $i \in \mathcal{H}_{k}$, at time $t=0$. Knowing the available link capacities $\mathcal{C}^{j}$ and link prices $\mathcal{P}^{j}$ for $j \in \mathcal{G}_{i}$, each lower tier ISP $i$ calculates its optimal routing (traffic transmission rates vectors) with the algorithm presented in Section III. The lower tier ISP sends the link bandwidth consumption, $z_{i}^{j}$, back to each higher tier ISP $j \in \mathcal{G}_{i}$. We call the feedback information $z_{i}^{j}$ as bidding of lower tier ISP $i$ to higher tier ISP $j$. Higher tier ISP $k$ receives all the biddings from its connected lower tier ISPs within a period of time $T$. At the end of each period, higher tier ISP $k$ recomputes the link resource allocation and sends the new allocation $\mathcal{C}_{i}^{k}$ to each connected lower tier ISP $i$, where $i \in \mathcal{H}_{k}$. Based on the new bandwidth allocation, 
each lower tier ISP calculates its optimal routing again and the process repeats itself. Note that there are several advantages for this framework. First, all the information that a lower tier ISP $i$ requires are the unit prices $p_{i j}$ 's and capacities $c_{i j}$ 's of its private links, and the allocated link capacities $\mathcal{C}_{i}^{j}$ and prices $\mathcal{P}^{j}$ for $j \in \mathcal{G}_{i}$. These can be viewed as the private information of lower tier ISP $i$. Lower tier ISP $i$ does not have to know the bandwidth allocations $\left(\mathcal{C}_{1}^{j}, \ldots, \mathcal{C}_{n}^{j}\right)$ and bandwidth consumptions $\left(z_{1}^{k}, \ldots, z_{n}^{k}\right)$ since they are confidential information. Secondly, when the higher tier ISP $k$ makes the bandwidth allocation, it only has to know the biddings $\left(z_{1}^{k}, z_{2}^{k}, \ldots, z_{n}^{k}\right)$ from its connected ISPs and it needs not know the utility functions and the pricing information of the private peering links. Thirdly, the overhead of exchanging the control information in the framework is very small.

The distributed resource allocation algorithm is called the Equal Share Algorithm. At each round, a higher tier ISP distributes its "remaining" capacity equally among all lower tier ISPs after satisfying their bandwidth consumption demands indicated by their biddings. Initially, each higher tier ISP $k$ for $k \in\{1,2, \ldots, m\}$ allocates its link capacity equally to every lower tier ISP, i.e., $\mathcal{C}_{i}^{k}=\frac{n_{k} \mathcal{C}^{k}}{n_{k}}=\mathcal{C}^{k}$ and sends the capacity allocation $\mathcal{C}_{i}^{k}$ to every lower tier ISP $i$ for all $i \in \mathcal{H}_{k}$, Then each lower tier ISP applies the algorithm proposed in Section III to find its optimal bandwidth consumption (i.e., $z_{i}^{k}$ for lower tier ISP $i$ ) and sends the information back to the higher tier ISP $k$ as its resource bidding. When a higher tier ISP gathers all the feedbacks from its connected ISPs, it allocates to each lower tier ISP the capacity it bids, and then it allocates the remaining resource equally to them. Formally, the allocation is $\mathcal{C}_{i}^{k}=z_{i}^{k}+\frac{n_{k} \mathcal{C}^{k}-\bar{z}^{k}}{n_{k}}$. The ESA algorithm is:

\section{Equal Share Algorithm:}

1. Higher tier ISP $k$ initiates $\mathcal{C}_{i}^{k(0)}:=\frac{n_{k} \mathcal{C}^{k}}{n_{k}}:=\mathcal{C}^{k}$ to each lower tier ISP $i \in \mathcal{H}_{k}$. Set counter $t:=0$.

2. while (TRUE) \{

3. Higher tier ISP $k$ passes $\mathcal{C}_{i}^{k(t)}$ to each lower tier ISP $i$ for $i \in \mathcal{H}_{k}$;

4. for $\left(i \in \mathcal{H}_{k}\right)\{$

5. Lower tier ISP $i$ computes $\vec{y}_{i}^{k(t)}$ and $\vec{z}_{i}^{k(t)}$ with the algorithms in Sec. III;

and sends $z_{i}^{k(t)}=\sum_{j \in \mathcal{T}_{i}} z_{i j}^{k(t)}$ back to higher tier ISP $k$;

6. $\} / *$ termination of for-loop */

7. Higher tier ISP $k$ updates $\mathcal{C}_{i}^{k(t+1)}:=z_{i}^{k(t)}+\frac{n_{k} \mathcal{C}^{k}-\bar{z}^{k(t)}}{n_{k}}$ for every lower tier ISP $i \in \mathcal{H}_{k}$;

update counter $t:=t+1$

8. $\} / \star$ termination of while-loop */

Illustration of the ESA: To illustrate the performance of ESA, we carry out experiments and demonstrate the bandwidth allocation under two different scenarios: (i) the network has sufficient bandwidth capacity, and (ii) the network has insufficient bandwidth capacity.

Experiment IV-1 illustrates when the network has sufficient bandwidth resource. There are two higher tier ISPs and three lower tier ISPs in the network. Each lower tier ISP has two private peering links 


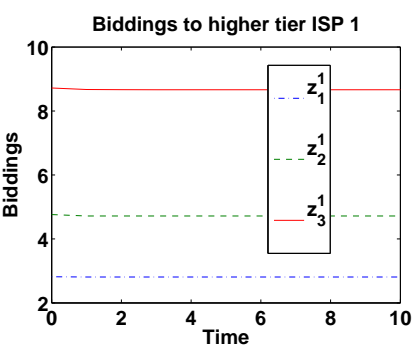

(a)

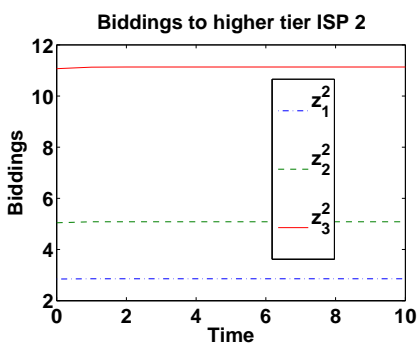

(d)

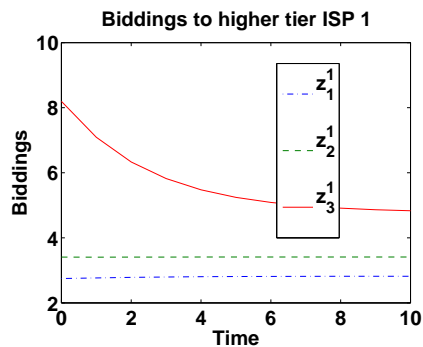

(b)

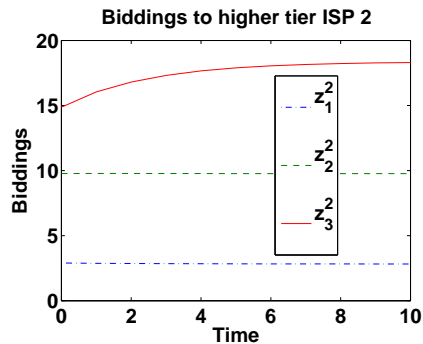

(e)

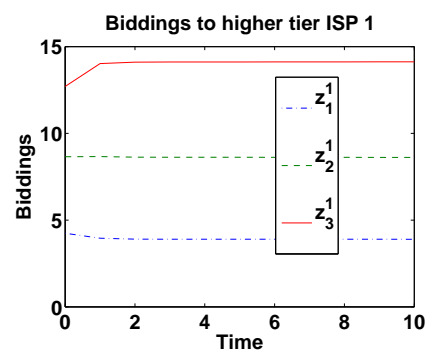

(c)

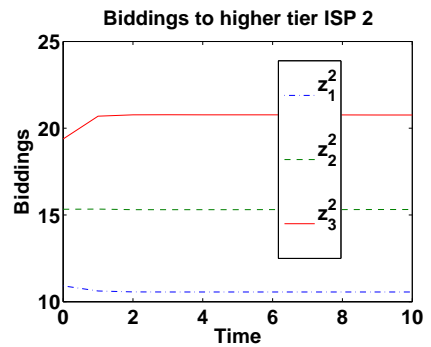

(f)

Fig. 3. Experiment IV-1: Biddings of lower tier ISPs 1,2 and 3 under sufficient bandwidth scenario, (a)-(c) are biddings to higher tier ISP 1 when $\gamma=1,5$ and 50,(d)-(f) are biddings to higher tier ISP 2 when $\gamma=1,5$ and 50 .

connecting to other lower tier ISPs with capacity $c_{i j}=10$ and unit price $p_{i j}=1$. Lower tier ISPs 1 , 2 and 3 have different values of happiness weighting coefficients, $w_{1 j}=10, w_{2 j}=15$ and $w_{3 j}=20$ for $j=1,2,3$. Higher tier ISP 1 provides a link with capacity $n_{1} \mathcal{C}^{1}=100$ and charges a unit price of $\mathcal{P}^{1}=1.5$. Higher tier ISP 2 provides a link with capacity $n_{2} \mathcal{C}^{2}=120$ and charges the same unit price of $\mathcal{P}^{2}=1.5$. Both higher tier ISPs update the distributions and send signals to lower tier ISPs every one second. Then each lower tier ISP computes its own optimal routing based on the method proposed in section III. Figure 3 shows the biddings of lower tier ISP to higher tier ISPs 1 and 2 throughout the experiment with different values of $\gamma$. The vertical axis shows the biddings of each lower tier ISP and the horizontal axis shows the time. For larger value of $\gamma$, the lower tier ISPs have larger concern in the QoS (congestion cost) of the links. They prefer to pay more for better transit service and they choose to send the traffic through the links of the higher tier ISPs, in which the congestion costs are lower. As a result, the lower tier ISPs give larger biddings. A point to note is that the utilities of lower tier ISPs are actually decreasing even they give larger biddings to the higher tier ISPs. The figures also show that the biddings of the lower tier ISPs converge in only a few intervals.

Experiment IV-2 illustrates the case when the network has insufficient bandwidth resource. The settings are the same as that of Experiment IV-1 but the happiness weighting coefficients. We set $w_{1 j}=100$, $w_{2 j}=150$ and $w_{3 j}=200$ for $j=1,2,3$. Note that the larger values of happiness weighting coefficients here means that lower tier ISPs are now having much stronger desires to transmit traffic. Thus keeping the network capacities at the same level leads to an insufficient bandwidth resource supply. Figure 4 


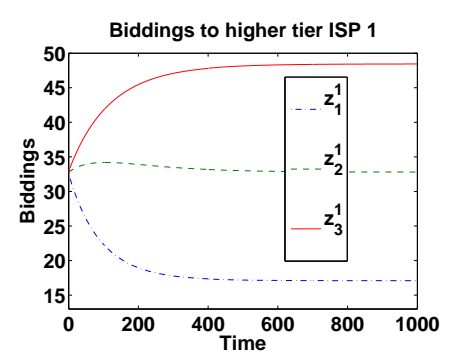

(a)

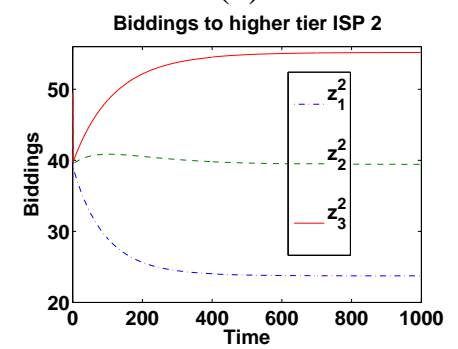

(d)

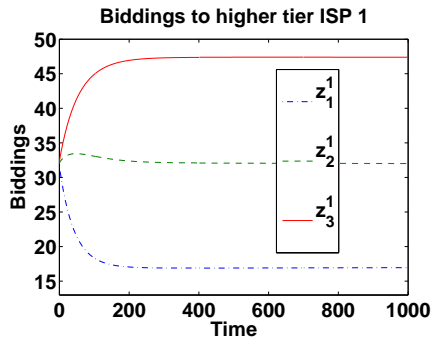

(b)

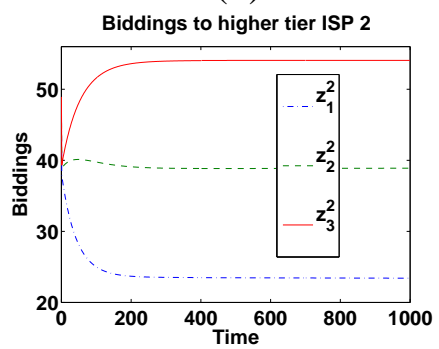

(e)

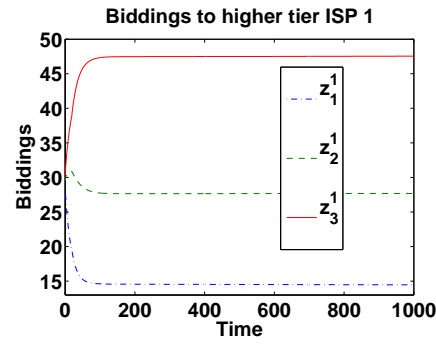

(c)

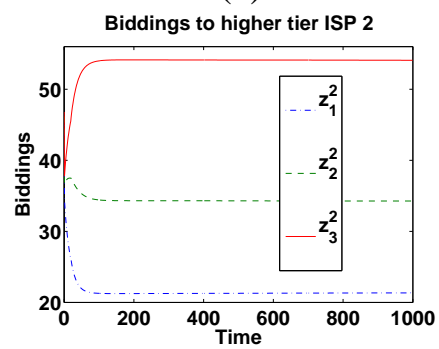

(f)

Fig. 4. Experiment IV-2: Biddings of lower tier ISPs 1,2 and 3 under insufficient bandwidth scenario, (a)-(c) are biddings to higher tier ISP 1 when $\gamma=1,5$ and 50, (d)-(f) are biddings to higher tier ISP 2 when $\gamma=1,5$ and 50.

shows the biddings of each lower tier ISP to higher tier ISPs 1 and 2 throughout the experiment with different values of $\gamma$. The figure shows that the biddings of lower tier ISPs converge even in insufficient resource scenario, but it takes more interval to converge than the previous experiment. The value of $\gamma$ does not change the convergence point of the biddings.

From these experiments, we have the following observations. Firstly, all the biddings of lower tier ISPs 1, 2 and 3 decrease with increasing value of $\gamma$. This is due to the larger concern in QoS of the higher tier ISPs' links. Secondly, the convergence rate of biddings is faster for larger value of $\gamma$. The most important observation is that monopolization of resource does not occur.

\section{Profit Maximization for Higher Tier ISPs}

For the higher tier ISPs, the most important issue is how to maximize one's profit. The profit of a higher tier ISP is the total payments received from its connected lower tier ISPs. One can express the profit maximization as:

$$
\mathcal{R}^{k}\left(\mathcal{P}^{k}\right)=\sum_{i \in \mathcal{H}_{k}} \mathcal{P}^{k} \cdot z_{i}^{k}\left(\mathcal{P}^{k}\right)=\mathcal{P}^{k} \sum_{i \in \mathcal{H}_{k}} z_{i}^{k}\left(\mathcal{P}^{k}\right)
$$

The notation $z_{i}^{k}\left(\mathcal{P}^{k}\right)$ represents the bandwidth consumption on higher tier ISP $k$ by a lower tier ISP and it is a function of the unit price $\mathcal{P}^{k}$ which is decided by the higher tier ISP $k$. If the price $\mathcal{P}^{k}$ is set too high, the lower tier ISPs may switch their traffic to the other higher tier ISPs (this is market competition), or to their private peering links for cheaper transit service. On the other hand, a lower price attracts lower tier 
ISPs for transit service but setting too low a price causes a decrease in profit. The dependency between unit price and profit is an interesting and important question for higher tier ISP: how to search for an optimal price to maximize its revenue?

In this paper, we propose an efficient method to estimate the price. This method requires a quick estimation of the aggregate bandwidth consumption $\bar{z}^{k}\left(\mathcal{P}^{k}\right)$ for a fixed unit price $\mathcal{P}^{k}$. With the estimate of $\bar{z}^{k}\left(\mathcal{P}^{k}\right)$, one can easily calculate the profit of the higher tier ISP $k$ by Eq.(12). Before we present the optimal price search method, let us first illustrate how a higher tier ISP $k$ can estimate the aggregate bandwidth consumption with a fixed unit price $\mathcal{P}^{k}$. The estimation has the following four assumptions:

A1. All higher tier ISPs apply the ESA proposed in Section IV in allocating the bandwidth resource. The reason is that ESA can avoid monopolization of bandwidth resource by some lower tier ISPs.

A2. Every higher tier ISP takes an indiscriminate pricing approach and charges the same unit price to all connected lower tier ISPs, i.e. $\mathcal{P}_{i}^{k}=\mathcal{P}^{k}$ for all $k \in\{1,2, \ldots, m\}$ and $i \in \mathcal{H}_{k}$, which means they are competing for the same set of customers.

A3. Every higher tier ISP is providing transit service for all $n$ lower tier ISPs.

A4. When higher tier ISP $k$ maximizes its profit, it only considers the case of $z_{i j}^{k}>0$ for all $i \in \mathcal{H}_{k}$, which means all lower tier ISPs transmit data via it.

\section{A. Estimation of aggregate bandwidth consumption $\bar{z}^{k_{1}}$}

To estimate the aggregate bandwidth consumption (or biddings), we introduce a variable $t^{k_{1}}$, which represents the marginal increase in congestion cost plus payment in transmitting data through higher tier ISP $k_{1}$. The purpose of introducing the variable $t^{k_{1}}$ is that one can represent the aggregate biddings $\bar{z}^{k_{1}}$ in terms of $t^{k_{1}}$. Given a unit price $\mathcal{P}^{k_{1}}$, to estimate the revenue $\mathcal{R}^{k_{1}}\left(\mathcal{P}^{k_{1}}\right)$, one can first estimate the value of $t^{k_{1}}$, then estimates the aggregate biddings $\bar{z}^{k_{1}}$ and revenue $\mathcal{R}^{k_{1}}\left(\mathcal{P}^{k_{1}}\right)$.

Under the ESA and at the equilibrium point of the biddings by lower tier ISPs, we have

$$
\mathcal{C}_{i}^{k}-z_{i}^{k}=\frac{n_{k} \mathcal{C}^{k}-\bar{z}^{k}}{n_{k}} \quad \text { for } i \in \mathcal{H}_{k} \text { and } k \in\{1,2, \ldots, m\}
$$

Substituting Eq.(13) into the necessary conditions in Eq.(11) and by A3, we have

$$
\begin{gathered}
\frac{w_{i j}}{1+y_{i j}+\sum_{k \in \mathcal{G}_{i}} z_{i j}^{k}} \leq \frac{\gamma}{\left(c_{i j}-y_{i j}\right)^{2}}+p_{i j}, \\
\frac{w_{i j}}{1+y_{i j}+\sum_{k \in \mathcal{G}_{i}} z_{i j}^{k}}=\frac{\gamma n_{k_{1}}^{2}}{\left(n_{k_{1}} \mathcal{C}^{k_{1}}-\bar{z}^{k_{1}}\right)^{2}}+\mathcal{P}^{k_{1}}, \quad \frac{w_{i i}}{1+\sum_{k \in \mathcal{G}_{i}} z_{i i}^{k}}=\frac{\gamma n_{k_{1}}^{2}}{\left(n_{k_{1}} \mathcal{C}^{k_{1}}-\bar{z}^{k_{1}}\right)^{2}}+\mathcal{P}^{k_{1}} \\
\frac{w_{i j}}{1+y_{i j}+\sum_{k \in \mathcal{G}_{i}} z_{i j}^{k}} \leq \frac{\gamma n_{k_{2}}^{2}}{\left(n_{k_{2}} \mathcal{C}^{k_{2}}-\bar{z}^{k_{2}}\right)^{2}}+\mathcal{P}^{k_{2}},
\end{gathered}
$$


Define the variable $t^{k_{1}}$, which is

$$
t^{k_{1}}=\frac{\gamma}{\left(\mathcal{C}_{i}^{k_{1}}-z_{i}^{k_{1}}\right)^{2}}+\mathcal{P}^{k_{1}}=\frac{\gamma n_{k_{1}}^{2}}{\left(n_{k_{1}} \mathcal{C}^{k_{1}}-\bar{z}^{k_{1}}\right)^{2}}+\mathcal{P}^{k_{1}}
$$

We can express $\bar{z}^{k_{1}}$ in terms of $t^{k_{1}}$ as

$$
\bar{z}^{k_{1}}=n_{k_{1}} \mathcal{C}^{k_{1}}-\frac{n_{k_{1}} \sqrt{\gamma}}{\sqrt{t^{k_{1}}-\mathcal{P}^{k_{1}}}}
$$

Now, we have three cases to consider: (1) when $z_{i j}^{k_{2}}>0$ and $y_{i j}>0$ (i.e., there are traffic in all other links), (2) when $z_{i j}^{k_{2}}>0$ and $y_{i j}=0$ (i.e., there are traffic through other higher tier ISPs and there is no traffic through private peering link), (3) when $z_{i j}^{k_{2}}=0$ (i.e., there is only one higher tier ISP $k_{1}$ in the network). Let us consider the case (1) first. The necessary conditions of Eq.(14), (15) and (16) become

$$
\begin{aligned}
z_{i j}^{k_{1}} & =\frac{w_{i j}}{t^{k_{1}}}-1-y_{i j}-\sum_{k \neq k_{1} \in \mathcal{G}_{i}} z_{i j}^{k}, \\
z_{i i}^{k_{1}} & =\frac{w_{i i}}{t^{k_{1}}}-1-\sum_{k \neq k_{1} \in \mathcal{G}_{i}} z_{i i}^{k}, \\
y_{i j} & =c_{i j}-\frac{\sqrt{\gamma}}{\sqrt{t^{k_{1}}-p_{i j}}}, \\
\bar{z}^{k_{2}} & =n_{k_{2}} \mathcal{C}^{k_{2}}-\frac{n_{k_{2}} \sqrt{\gamma}}{\sqrt{t^{k_{1}}-P^{k_{2}}}}
\end{aligned}
$$

Note that the requirement for $y_{i j}>0$ is $t^{k_{1}}>p_{i j}$ and that for $z_{i j}^{k_{2}}>0$ is $t^{k_{1}}>\mathcal{P}^{k_{2}}$. Substituting Eq.(19), (20), (21), (22) into $\bar{z}^{k_{1}}=\sum_{i \in \mathcal{H}_{k_{1}}} \sum_{j} z_{i j}^{k_{1}}$ and applying $\mathbf{A 3}$, we have:

$$
\begin{aligned}
& \bar{z}^{k_{1}}=\sum_{i} \sum_{j \neq i}\left(\frac{w_{i j}}{t^{k_{1}}}-1-y_{i j}-\sum_{k \neq k_{1}} z_{i j}^{k}\right)+\sum_{i}\left(\frac{w_{i i}}{t^{k_{1}}}-1-\sum_{k \neq k_{1}} z_{i i}^{k}\right) \\
& =\sum_{i} \sum_{j} \frac{w_{i j}}{t^{k_{1}}}-\sum_{i} \sum_{j} 1-\sum_{i} \sum_{j} \sum_{k \neq k_{1}} z_{i j}^{k}-\sum_{i} \sum_{j \neq i} y_{i j} \\
& =\sum_{i} \sum_{j} \frac{w_{i j}}{t^{k_{1}}}-n^{2}-\sum_{k \neq k_{1}} \bar{z}^{k}-\sum_{i} \sum_{j \neq i} y_{i j} \\
& =\sum_{i} \sum_{j} \frac{w_{i j}}{t^{k_{1}}}-n^{2}-\sum_{k \neq k_{1}}\left(n \mathcal{C}^{k}-\frac{n \sqrt{\gamma}}{\sqrt{t^{k_{1}}-\mathcal{P}^{k}}}\right)-\sum_{i} \sum_{j \neq i}\left(c_{i j}-\frac{\sqrt{\gamma}}{\sqrt{t^{k_{1}}-p_{i j}}}\right) \\
& \bar{z}^{k_{1}}=\frac{\bar{W}}{t^{k_{1}}}-n^{2}-\sum_{k \neq k_{1}} n \mathcal{C}^{k}-\sum_{i} \sum_{j \neq i} c_{i j}+\sum_{k \neq k_{1}} \frac{n \sqrt{\gamma}}{\sqrt{t^{k_{1}}-\mathcal{P}^{k}}}+\sum_{i} \sum_{j \neq i} \frac{\sqrt{\gamma}}{\sqrt{t^{k_{1}}-p_{i j}}}
\end{aligned}
$$


In here, $\bar{W}$ is the sum of happiness weighting coefficients of lower tier ISPs $\left(\bar{W}=\sum_{i} \sum_{j} w_{i j}\right)$. Eq.(18) and (23), we have

$$
\begin{aligned}
n \mathcal{C}^{k_{1}}+\sum_{k \neq k_{1}} n \mathcal{C}^{k}+\sum_{i} \sum_{j \neq i} c_{i j}+n^{2} & =\frac{\bar{W}}{t^{k_{1}}}+\sum_{k} \frac{n \sqrt{\gamma}}{\sqrt{t^{k_{1}}-\mathcal{P}^{k}}}+\sum_{i} \sum_{j \neq i} \frac{\sqrt{\gamma}}{\sqrt{t^{k_{1}}-p_{i j}}} \\
\overline{\mathcal{C}}_{0}+n^{2} & \approx \frac{\bar{W}}{t^{k_{1}}}+\sum_{k} \frac{n \sqrt{\gamma}}{\sqrt{t^{k_{1}}-\mathcal{P}^{k}}}+\frac{n(n-1) \sqrt{\gamma}}{\sqrt{t^{k_{1}}-p_{a v}}}
\end{aligned}
$$

where $p_{a v}=\frac{1}{n(n-1)} \sum_{i} \sum_{j \neq i} p_{i j}$ is the mean of all $p_{i j}$ s and $\overline{\mathcal{C}}_{0}=n \mathcal{C}^{k_{1}}+\sum_{k \neq k_{1}} n \mathcal{C}^{k}+\sum_{i} \sum_{j \neq i} c_{i j}$ is the aggregate capacities in the system. When the variance of the unit price of peering link is small, one can derive the estimate of Eq.(24).

Once a higher tier ISP has the information of the happiness weighting coefficients, the capacities in the system, and unit prices of the private links and links of other higher tier ISPs, then the higher tier ISP $k_{1}$ can compute the value of $t^{k_{1}}$ using Eq.(24) and it can estimate the aggregate biddings $\bar{z}^{k_{1}}$ with Eq.(18) and its profit $\mathcal{R}^{k_{1}}\left(\mathcal{P}^{k_{1}}\right)$ using Eq.(12).

Consider case (2) where $y_{i j}=0$ for $j \neq i$ and $z_{i j}^{k_{2}}>0$ for $k_{2} \neq k_{1}$ and $j \in\{1,2, \ldots, n\}$. The requirement for Eq.(14) and (16) to hold are:

$$
t^{k_{1}}=\frac{w_{i j}}{1+\sum_{k \in \mathcal{G}_{i}} z_{i j}^{k}} \leq \frac{\gamma}{c_{i j}^{2}}+p_{i j} \text { and } t^{k_{1}}>\mathcal{P}^{k_{2}}
$$

Substituting Eq.(19), (20), (22) into $\bar{z}^{k_{1}}=\sum_{i \in \mathcal{H}_{k}} \sum_{j} z_{i j}^{k_{1}}$ and applying A3, we have:

$$
\begin{aligned}
\bar{z}^{k_{1}} & =\sum_{i} \sum_{j}\left(\frac{w_{i j}}{t^{k_{1}}}-1-\sum_{k \neq k_{1}} z_{i j}^{k}\right)=\sum_{i} \sum_{j} \frac{w_{i j}}{t^{k_{1}}}-\sum_{i} \sum_{j} 1-\sum_{k \neq k_{1}} \bar{z}^{k} \\
& =\sum_{i} \sum_{j} \frac{w_{i j}}{t^{k_{1}}}-n^{2}-\sum_{k \neq k_{1}}\left(n \mathcal{C}^{k}-\frac{n \sqrt{\gamma}}{\sqrt{t^{k_{1}}-\mathcal{P}^{k}}}\right) .
\end{aligned}
$$

Equating Eq.(18) and (25), we have

$$
\begin{aligned}
n \mathcal{C}^{k_{1}}-\frac{n \sqrt{\gamma}}{\sqrt{t^{k_{1}}-\mathcal{P}^{k_{1}}}} & =\sum_{i} \sum_{j} \frac{w_{i j}}{t^{k_{1}}}-n^{2}-\sum_{k \neq k_{1}} n \mathcal{C}^{k}+\sum_{k \neq k_{1}} \frac{n \sqrt{\gamma}}{\sqrt{t^{k_{1}}-\mathcal{P}^{k}}} \\
n \mathcal{C}^{k_{1}}+\sum_{k \neq k_{1}} n \mathcal{C}^{k}+n^{2} & =\frac{\bar{W}}{t^{k_{1}}}+\frac{n \sqrt{\gamma}}{\sqrt{t^{k_{1}}-\mathcal{P}^{k_{1}}}}+\sum_{k \neq k_{1}} \frac{n \sqrt{\gamma}}{\sqrt{t^{k_{1}}-\mathcal{P}^{k}}} .
\end{aligned}
$$

Once again, higher tier ISP $k_{1}$ can compute the value of $t^{k_{1}}$ with Eq.(26), then apply Eq.(18) to find the total biddings and Eq.(12) to find its profit. 
For case (3) when $z_{i j}^{k_{2}}=0$ for $k_{2} \neq k_{1}$, it is just like the situation where there is only one higher tier ISP $k_{1}$ in the network providing transit service. So higher tier ISP $k_{1}$ can apply

$$
\begin{aligned}
n \mathcal{C}^{k_{1}}+\sum_{i} \sum_{j \neq i} c_{i j}+n^{2} & \approx \frac{\bar{W}}{t^{k_{1}}}+\frac{n \sqrt{\gamma}}{\sqrt{t^{k_{1}}-\mathcal{P}^{k_{1}}}}+\frac{n(n-1) \sqrt{\gamma}}{\sqrt{t^{k_{1}}-p_{a v}}} \\
n \mathcal{C}^{k_{1}}+n^{2} & =\frac{\bar{W}}{t^{k_{1}}}+\frac{n \sqrt{\gamma}}{\sqrt{t^{k_{1}}-\mathcal{P}^{k_{1}}}}
\end{aligned}
$$

to estimate $t^{k_{1}}$, then use Eq.(18) to find the total biddings and Eq.(12) to find its profit.

\section{B. Optimal Pricing Search Method}

Let us first give the intuitive idea of our optimal pricing search method. When the unit price of a higher tier ISP $k$ is small, a small increase in the price only reduces the total biddings of the lower tier ISPs $\bar{z}^{k}$ slightly. So higher tier ISP $k$ should have an increase of profit as the loss in the decreasing bidding is covered by the gain in the increment of unit price. The increase of profit vanishes when the marginal point is reached.

Our pricing search method has two phases. In phase one, we obtain a feasible range of the optimum unit price with the help of the estimates of aggregate traffic in section V-A. Phase two aims at reducing the size of the feasible range obtained in phase one by trisection method. The pricing search method is:

\section{Pricing Search Method:}

1. Higher tier ISP initiates a step size $\sigma$ and a threshold $\delta$.

2. /* Phase 1: */

3. while (1) \{

4. Higher tier ISP computes four unit prices $\mathcal{P}_{1}=\sigma, \mathcal{P}_{2}=2 \mathcal{P}_{1}, \mathcal{P}_{3}=2 \mathcal{P}_{2}$ and $\mathcal{P}_{4}=2 \mathcal{P}_{3}$.

5. Higher tier ISP computes four revenues $\mathcal{R}\left(\mathcal{P}_{1}\right), \mathcal{R}\left(\mathcal{P}_{2}\right), \mathcal{R}\left(\mathcal{P}_{3}\right)$ and $\mathcal{R}\left(\mathcal{P}_{4}\right)$.

6. $\quad$ if $\left(\mathcal{R}\left(\mathcal{P}_{3}\right)>\mathcal{R}\left(\mathcal{P}_{4}\right)\right)$

7. break; $/ \star$ go to phase $2 \star /$

8. else

9. $\sigma=2 \sigma / \star$ go back to phase $1 * /$

10. \}/* termination of while-loop of phase 1 */

11. /* Phase 2: */

12. while (1) \{

13. if $\left(\mathcal{P}_{4}-\mathcal{P}_{1}<\delta\right)$

14. $\quad$ return $\mathcal{P}_{1}$; break;

15. $\quad$ else if $\left(\mathcal{R}\left(\mathcal{P}_{2}\right)<\mathcal{R}\left(\mathcal{P}_{3}\right)\right)$

16. update $\mathcal{P}_{1}=\mathcal{P}_{2}, \mathcal{P}_{2}=\mathcal{P}_{3}$ and $\mathcal{P}_{3}=\frac{\mathcal{P}_{2}+\mathcal{P}_{4}}{2}$.

17. else if $\left(\mathcal{R}\left(\mathcal{P}_{2}\right)>\mathcal{R}\left(\mathcal{P}_{3}\right)\right)$

18. update $\mathcal{P}_{4}=\mathcal{P}_{3}, \mathcal{P}_{3}=\mathcal{P}_{2}$ and $\mathcal{P}_{2}=\frac{\mathcal{P}_{1}+\mathcal{P}_{3}}{2}$.

19. \}/* termination of while-loop of phase 2 */ 
At the beginning, a higher tier ISP chooses a step size $\sigma$ and a threshold $\delta$. In phase one, the higher tier ISP finds four unit prices based on $\sigma$ such that $\mathcal{P}_{1}=\sigma, \mathcal{P}_{2}=2 \mathcal{P}_{1}, \mathcal{P}_{3}=2 \mathcal{P}_{2}$ and $\mathcal{P}_{4}=2 \mathcal{P}_{3}$. Then it estimates the profit at the four pricing levels, $\mathcal{R}\left(\mathcal{P}_{1}\right), \mathcal{R}\left(\mathcal{P}_{2}\right), \mathcal{R}\left(\mathcal{P}_{3}\right)$ and $\mathcal{R}\left(\mathcal{P}_{4}\right)$. The condition $\mathcal{R}\left(\mathcal{P}_{3}\right)>\mathcal{R}\left(\mathcal{P}_{4}\right)$ means that the feasible range $\left[\mathcal{P}_{1}, \mathcal{P}_{4}\right]$ is found and jump to phase two. Otherwise, if $\mathcal{R}\left(\mathcal{P}_{3}\right) \leq \mathcal{R}\left(\mathcal{P}_{4}\right)$, we update $\sigma=2 \sigma$ and go back to phase one again. The stopping criteria is based on the assumption that a local optimal price is also the global optimal price.

For phase two, the objective is to reduce the feasible range obtained in phase one to be within the threshold via the trisection method. We compare the two revenues $\mathcal{R}\left(\mathcal{P}_{2}\right)$ and $\mathcal{R}\left(\mathcal{P}_{3}\right)$. If $\mathcal{R}\left(\mathcal{P}_{2}\right) \leq$ $\mathcal{R}\left(\mathcal{P}_{3}\right)$, the optimum unit price is in between $\left[\mathcal{P}_{2}, \mathcal{P}_{4}\right]$. This is again based on the assumption that a local optimal price is also the global optimal price. So we update $\mathcal{P}_{1}=\mathcal{P}_{2}$ and $\mathcal{P}_{2}=\mathcal{P}_{3}$ and $\mathcal{P}_{3}=\frac{\mathcal{P}_{2}+\mathcal{P}_{4}}{2}$. If $\mathcal{R}\left(\mathcal{P}_{2}\right)>\mathcal{R}\left(\mathcal{P}_{3}\right)$, the optimum unit price is in the range $\left[\mathcal{P}_{1}, \mathcal{P}_{3}\right]$, and we update $\mathcal{P}_{4}=\mathcal{P}_{3}, \mathcal{P}_{3}=\mathcal{P}_{2}$ and $\mathcal{P}_{2}=\frac{\mathcal{P}_{1}+\mathcal{P}_{3}}{2}$. Phase two ends when the size of the range, $\mathcal{P}_{4}-\mathcal{P}_{1}<\delta$ (the threshold).

In summary, we present a method for a higher tier ISP to quickly search for the optimal price, in which it obtains maximum revenue. Note that the optimal price $\mathcal{P}^{*}$ is computed based on the pricing levels of other higher tier ISPs.

\section{Illustration of Optimal Pricing Policy}

To illustrate the correctness and effectiveness of Eq.(24) and (26), we carry out two experiments to demonstrate how a higher tier ISP can find the optimal unit price. In Experiment V-1, we consider a network of two higher tier ISPs and five lower tier ISPs. Each lower tier ISP has four private peering links connecting to other lower tier ISPs with capacity $c_{i j}=10$ and unit price $p_{i j}=1$. The lower tier ISPs have different values of happiness weighting coefficients, $w_{1 j}=18, w_{2 j}=19, w_{3 j}=20, w_{4 j}=21$, $w_{5 j}=22$ for $j \in\{1, \ldots, 5\}$. Each higher tier ISP is providing transit service for all the lower tier ISPs. Higher tier ISP 1 provides a link with capacity $n_{1} \mathcal{C}^{1}=100$ and $\mathcal{P}^{1}=1$.6. Higher tier ISP 2 provides a link with capacity $n_{2} \mathcal{C}^{2}=120$. Both higher tier ISPs apply the ESA to do resource allocation. Each of them will send signals to lower tier ISPs and update the resource allocation every one second. We are now going to find the optimal price of higher tier ISP $2\left(\mathcal{P}^{2}\right)$. Figure 5 shows the revenue of ISP 2 with different values of unit price $\mathcal{P}^{2}$. The vertical axis shows the actual revenue and estimated revenue of ISP 2 while the horizontal axis shows its link unit price. The actual revenue is computed at the equilibrium

point when the biddings from the lower tier ISPs converge. The estimated revenue is computed using the optimal pricing search method proposed in Section V-B and Eq.(24). One can observe that both the proposed algorithm and equation can accurately find out the optimal unit price is at about $\mathcal{P}^{2 *}=1.50$.

In Experiment V-2, we want to see if the optimal price is dependent of the initial pricing value. The settings in this experiment are the same as those in Experiment V-1 but higher tier ISP 1 also searches 


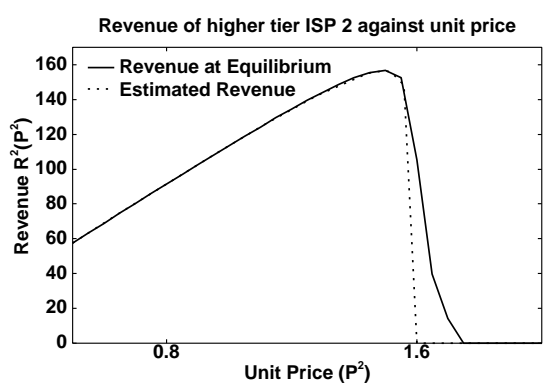

Fig. 5. Experiment V-1: Effectiveness of optimal pricing search method. The optimal price is at about 1.5.

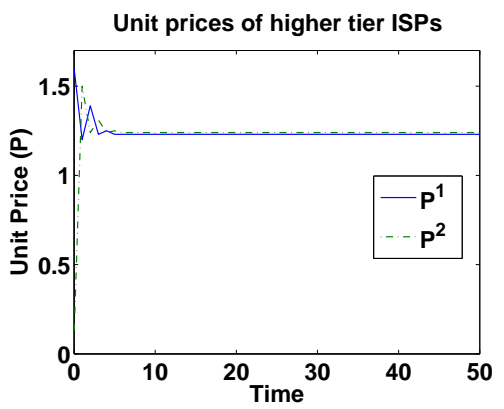

(a) Initial $\mathcal{P}^{2}=0.1$

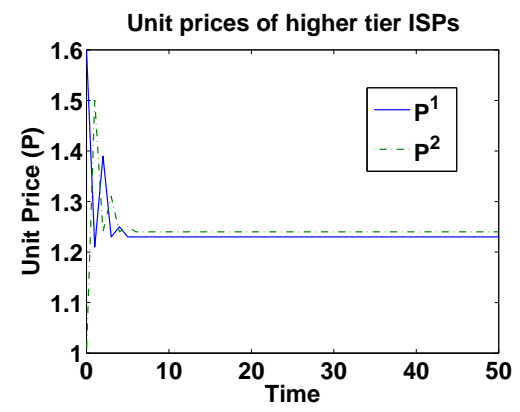

(b) Initial $\mathcal{P}^{2}=1.0$

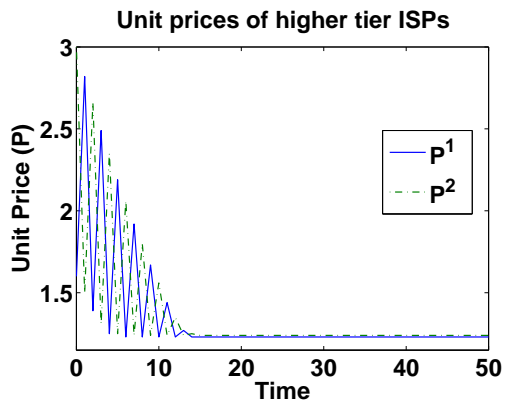

(c) Initial $\mathcal{P}^{2}=3.0$

Fig. 6. Experiment V-2: Convergence of Unit Prices. The optimal prices are 1.23 and 1.24.

for its optimal price with higher tier ISP 2. The current decision of the optimal price of one higher tier ISP depends on the previous announced unit price of the other. Higher tier ISP 1 has an initial price $\mathcal{P}^{1}=1.6$ and higher tier ISP 2 has an initial price $\mathcal{P}^{2}=0.1,1.0$ or 3.0. In each period, each higher tier ISP computes its optimal unit price based on the happiness coefficients of lower tier ISPs, aggregate capacities in the network, average unit price of peering links, as well as the unit price of the other higher tier ISP. Each higher tier ISP also computes the resource allocation for the lower tier ISPs. Figure 6 shows the optimal unit prices of the two higher tier ISPs during the experiment. We observed that the same final optimal prices $\mathcal{P}^{1}=1.23$ and $\mathcal{P}^{2}=1.24$ are computed, which is independent of the initial unit price of ISP 2.

In summary, we present the procedure for a higher tier ISP to estimate the total bandwidth consumption from its customers and also its revenue, with a fixed unit price on its link, and we show that the estimate and optimal pricing search method are efficient and the result is independent of initial values.

Lemma 1: The final converged price vectors computed by the optimal pricing search method is the Nash Equilibrium of the pricing game.

Proof: Let $\mathcal{P}^{-j}=\left(\mathcal{P}^{1}, \ldots, \mathcal{P}^{j-1}, \mathcal{P}^{j+1}, \ldots, \mathcal{P}^{m}\right)$ denote the price strategies of all higher tier ISPs except ISP $j$. When higher tier ISP $k_{1}$ searches for its optimal price $\mathcal{P}^{k_{1}}$, the estimations of $\sum_{k \neq k_{1}} n \mathcal{C}^{k}$, 
$\sum_{i} \sum_{j \neq i} c_{i j}$ and $p_{a v}$ are unchanged, so the solutions of $t^{k_{1}}$ of Eq.(24) and (26) are a function of $\mathcal{P}^{k_{1}}$ and $\mathcal{P}^{-k_{1}}$. For consistent strategies of other higher tier ISPs $\mathcal{P}^{-k_{1}}$, the optimal price $\mathcal{P}^{k_{1}}$ is unique. Therefore, the price vectors computed by the optimal pricing search method is the Nash Equilibrium of the price game.

\section{Performance under Network Scaling}

Let us consider the performance of the network when we scale up the number of ISPs. When there are more lower tier ISPs, the demand in transmitting through higher tier ISPs increases. On the other hand, the link capacity allocated to each lower tier ISP becomes smaller, which reduces the QoS guarantee. One important question for a higher tier ISP is that if it can get more profit (or increased revenue) by performing network upgrade? Furthermore, other higher tier ISPs may also upgrade their capacities and there are more private peering links among the lower tier ISPs. These factors make it complicated to decide whether one should perform network upgrade.

Our investigation is built on the results obtained in Section V. Eq.(24) and (26) provide an estimate of the aggregate bandwidth consumption on the transit service of a higher tier ISP. If a higher tier ISP knows some information of the network environment, e.g., estimates of the aggregate happiness coefficients of its customers, pricing policies and link capacities of the peering links and other higher tier ISPs, then it has an opportunity to infer its optimal pricing strategy to maximize its revenue. In general, the pricing policies and capacities of private peering links and other higher tier ISPs are regarded as confidential information which is difficult to obtain, yet rough estimates of them allow a higher tier ISP to make the its marketing decision. Another utility of Eq.(24) and (26) are to predict how the number of lower tier ISPs affects the maximum revenue of a higher tier ISP at its optimal pricing. This provides an important insight as we scale up the network. In the following, we will consider under two different situations: 1) there is no peering link between lower tier ISPs, and 2) lower tier ISPs set up peering relationships in a meshed peering manner.

\section{A. Network Scaling without peering links among lower tier ISPs}

As lower tier ISPs have only links connecting to higher tier ISPs, they must transmit through those links to communicate with each other. From the analysis in Section V, we know that when there is no peering link, i.e. $y_{i j}=0$, Eq.(26) holds and it can be approximated as:

$$
n \mathcal{C}^{k_{1}}+\sum_{k \neq k_{1}} n \mathcal{C}^{k}+n^{2}=\frac{n^{2} \bar{w}}{t^{k_{1}}}+\frac{n \sqrt{\gamma}}{\sqrt{t^{k_{1}}-\mathcal{P}^{k_{1}}}}+\sum_{k \neq k_{1}} \frac{n \sqrt{\gamma}}{\sqrt{t^{k_{1}}-\mathcal{P}^{k}}}
$$


wherein $\bar{w}$ is the average happiness coefficient of all lower tier ISPs. The condition for Eq.(26) to hold are $\mathcal{P}^{k}<t^{k_{1}} \leq \frac{\gamma}{c_{i j}^{2}}+p_{i j}$ for all $i, j$. This condition represents when there is no private peering link (i.e., $c_{i j}=0$ ), or the congestion cost plus payment to transmit data in peering link is too expensive. Applying the optimal pricing search method in Section V-B, the maximum revenue of higher tier ISP $k_{1}$ can be calculated under different values of $m$ and $n$. Figure 7 illustrates the maximum revenue of higher tier ISP $k_{1}$ against the number of lower tier ISPs $n$, for different numbers of higher tier ISPs $m$ in the network. In this figure, all the $m$ higher tier ISPs do not perform network upgrade even when there are increasing number of lower tier ISPs joining the network. So we keep $n \mathcal{C}^{k}=100$ for all $k \in\{1,2, \ldots, m\}$.

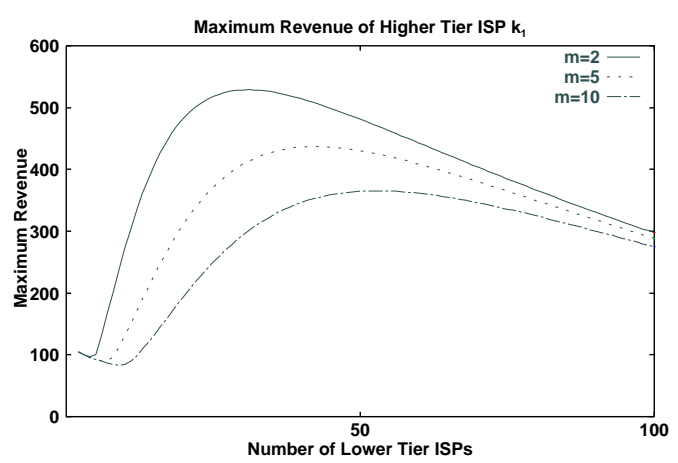

Fig. 7. Experiment VI-A-1 (without peering links): Higher tier ISP $k_{1}$ 's revenue against number of lower tier ISPs, with different number of higher tier ISPs in the network. All the link capacities of higher tier ISPs are kept constant: $n \mathcal{C}^{k}=100$ for all $k$, and $\bar{w}=10$.

Some important observations can be made. First, for a particular value of $m$, one can determine a unique number of lower tier ISPs $n^{*}$ that maximizes the revenue of higher tier ISP $k_{1}$. Further increase of lower tier ISPs will only decrease the ISP $k_{1}$ 's revenue. The justification is because the link bandwidth capacity of ISP $k_{1}$ allocated to each lower tier ISP is decreasing, which leads to an increasing congestion cost and so a decrease in the traffic demand. Also, when the number of higher tier ISPs $(m)$ increases, it needs more lower tier ISPs $(n)$ for higher tier ISP $k_{1}$ to obtain its maximum revenue. This is because the more lower tier ISPs increases the traffic demand in the network. Another observation is that more higher tier ISPs exist in the network reduces the revenue of ISP $k_{1}$.

Figure 8 illustrates Experiment VI-A-2 when the higher tier ISP $k_{1}$ 's link capacity increases proportionally to the number of lower tier ISPs in the network. Higher tier ISP $k_{1}$ has a total capacity of $n \mathcal{C}^{k_{1}}$ where $\mathcal{C}^{k_{1}}=100$ is a constant. The other higher tier ISPs have link unit price $\mathcal{P}^{k}=1.2$ and do not perform network upgrade and have fixed capacities, i.e. $n \mathcal{C}^{k}=100$ for $k \neq k_{1}$. Figure 8 (a) illustrates the maximum revenue of higher tier ISP $k_{1}$ as a function of the number of lower tier ISPs, with different number of higher tier ISPs in the network. The figure shows that when higher tier ISP $k_{1}$ upgrades its link capacity, no matter how many other higher tier ISPs are, it can always achieve an increasing revenue 
as $n$ increases, which is a contrast to the fixed-capacity case. Furthermore, the revenue is able to increase faster than $n$ does. Figure 8(b) shows the relationship between higher tier ISP $k_{1}$ 's maximal revenue per bandwidth $\left(\bar{z}^{k_{1}} \mathcal{P}^{k_{1} *} / n \mathcal{C}^{k_{1}}\right)$ and the number of lower tier ISPs, with different number of higher tier ISPs. It shows that higher tier ISP $k_{1}$ 's maximal revenue per bandwidth keeps increasing as $n$ grows, which implies that the higher tier ISP $k_{1}$ can benefit from upgrading its network.

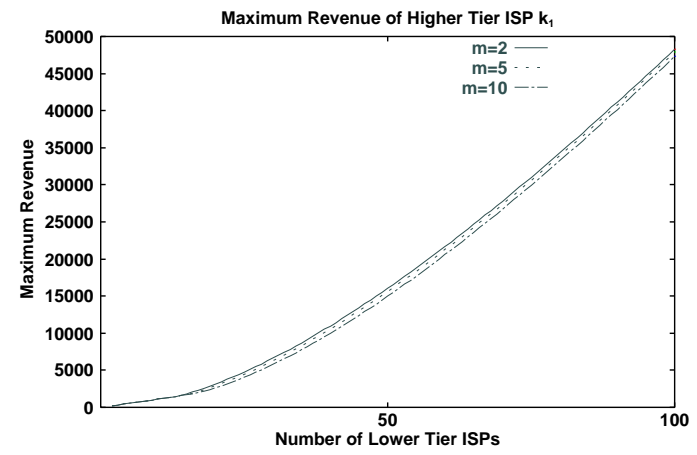

(a)

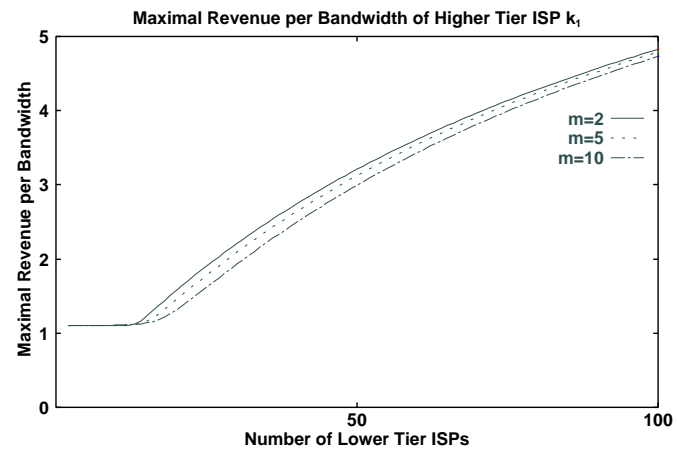

(b)

Fig. 8. Experiment VI-A-2 (without peering links): higher tier ISP $k_{1}$ 's revenue when the capacity of its link grows proportionally: (a) ISP $k_{1}$ 's maximum revenue v.s. $n$, (b) ISP $k_{1}$ 's maximal revenue per bandwidth v.s. $n$.

Lastly, we consider when all higher tier ISPs perform network upgrade. In Experiment VI-A-3, the link capacities of all higher tier ISPs are increasing proportional to the number of lower tier ISPs in the network. Thus every higher tier ISP $k$ has a total capacity of $n \mathcal{C}^{k}$ where $\mathcal{C}^{k}=100$ is a constant. Figure 9(a) illustrates higher tier ISP $k_{1}$ 's maximum revenue as a function of the number of lower tier ISPs, with different number of higher tier ISPs in the network. The figure shows if higher tier ISP $k_{1}$ upgrades its link capacity as other higher tier ISPs do, it can always achieve an increasing revenue as $n$ increases, and this result is independent on the number of higher tier ISPs in the network. Figure 9(b) shows the relationship between higher tier ISP $k_{1}$ 's maximal revenue per bandwidth and the network size $n$ with different values of $m$. Although the increasing rate of maximal revenue per bandwidth becomes slower when $m$ is larger, the marginal profit of higher tier ISP $k_{1}$ still does not decrease as more lower tier ISPs are joining the network, which implies that the higher tier ISP $k_{1}$ can benefit from upgrading its network.

\section{$B$. Network scaling with private peering links among lower tier ISPs}

Since there are private peering links, traffic can go through the peering link if the peering link provides a certain level of QoS and the price in transmitting is not high. With the existence of private peering links, we investigate whether there is any incentive for higher tier ISPs to perform network upgrade. In Section V, we showed that Eq.(24) holds if $y_{i j}>0$. The conditions for $y_{i j}>0$ holds are $t^{k_{1}} \geq \frac{\gamma}{c_{i j}^{2}}+p_{i j}$ and 


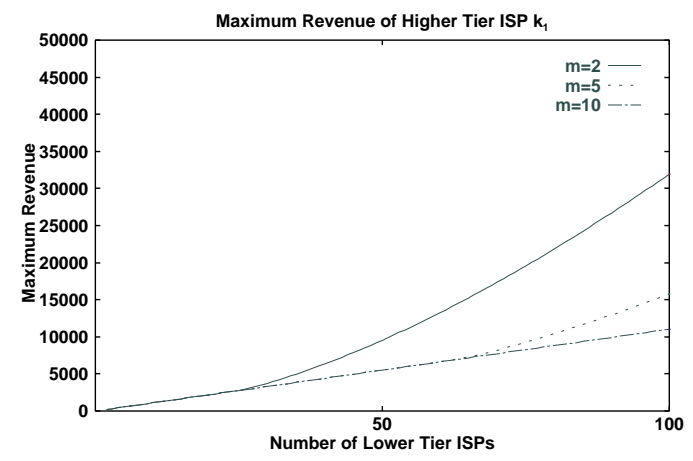

(a)

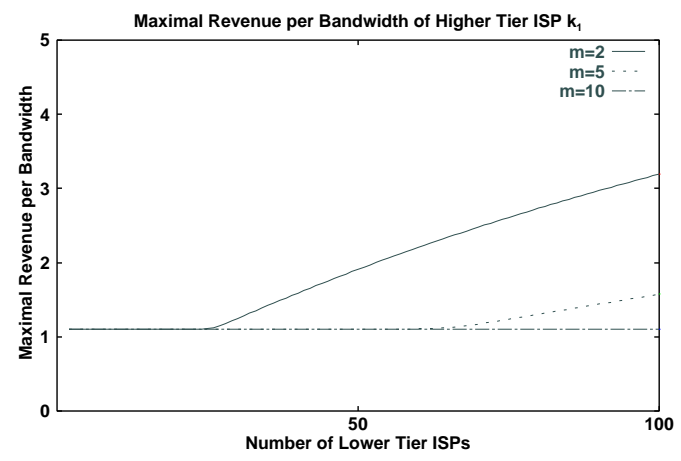

(b)

Fig. 9. Experiment VI-A-3 (without peering links): higher tier ISP $k_{1}$ 's revenue when the link capacities of all higher tier

ISPs grow proportionally: (a) ISP $k_{1}$ 's maximum revenue v.s. $n$, (b) ISP $k_{1}$ 's maximal revenue per bandwidth v.s. $n$.

$w_{i j}>t_{k_{1}}>\mathcal{P}^{k_{1}}+\frac{n^{2} \gamma}{\left(\mathcal{C}^{k_{1}}\right)^{2}}$. By solving Eq.(24), one can calculate the higher tier ISP $k_{1}$ 's maximum revenue for different values of $n$ and $m$. When a higher tier ISP $k$ upgrades its link bandwidth proportional to the number of lower tier ISPs $n$, its link capacity is $n \mathcal{C}^{k}$ where $\mathcal{C}^{k}=100$ is constant. When it does not perform upgrade, its link capacity $n \mathcal{C}^{k}=100$ is constant. We perform the analysis under the settings $m=10$ and $\mathcal{P}^{k}=0.8$ for all $k \neq k_{1}, w_{i j}=10$ for all $i, j$ and $p_{i j}=1, c_{i j}=10$ for all lower tier ISPs $j \neq i$. Figure 10 illustrates how the number of lower tier ISPs affects the maximum revenue of higher tier ISP $k_{1}$. The y-axis shows the maximal revenue per bandwidth of higher tier ISP $k_{1}$ when there is no higher tier ISP upgrades link capacity, or when only higher tier ISP $k_{1}$ upgrades link capacity, or when all $m$ higher tier ISPs do network upgrade. The horizontal axis shows the number of lower tier ISPs in the network.

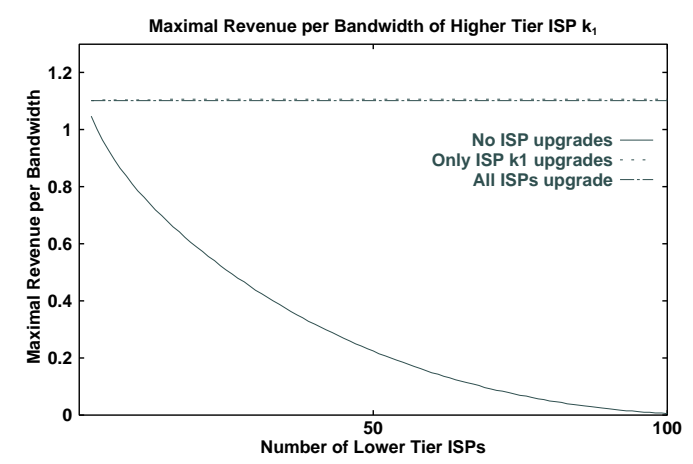

Fig. 10. Experiment VI-B (with peering links): higher tier ISP $k_{1}$ 's maximal revenue per bandwidth against $n$ under three situations: (a) no higher tier ISP upgrades, (b) only higher tier ISP $k_{1}$ upgrades, (c) all higher tier ISPs upgrade.

When the private peering links are introduced, if no higher tier ISP upgrades link capacity, increasing number of lower tier ISPs will lead to the decrease of revenue of all higher tier ISPs. This is caused by the increasing congestion cost in transmitting traffic through the higher tier ISP as link capacity shared becomes less. So lower tier ISPs opt to transmit through their own peering links. If only higher tier ISP 
$k_{1}$ upgrades link capacity, the lower tier ISPs still do not transmit traffic through the other higher tier ISPs due to the large congestion cost. However, they will send traffic through higher tier ISP $k_{1}$ as the congestion cost is small. So there is an increase in the maximal revenue per bandwidth when more lower tier ISPs join the network. Lastly, when all higher tier ISPs perform network upgrade, the maximum revenue of higher tier ISP $k_{1}$ can still increase and the rate of maximal revenue per bandwidth does not drop. For all scenarios, when compared to the case without peering links, the revenue increasing rate becomes much slower. This is due to the fact that lower tier ISPs have set up peering links to form a mesh-network. It is the competition that brings down the marginal profits of higher tier ISPs. It is worthwhile to mention that in practice, it may be difficult, or even impossible, for all lower tier ISPs to form a fully meshed network among themselves. This may be due to the geographical constraint that some of them are located very far apart, or may be due to the legal regulations. Thus there are still great opportunities for higher tier ISPs to gain by upgrading the network infrastructures.

\section{Related Work}

Let us present a brief review of some related work. In [4], authors study a cost model for peers in deciding whether to join a P2P network. There are some work on the game-theoretic study of ISP traffic[26] and overlay traffic interaction and sevice differentiation[8], [13] In [11], [14], [15], [17], [22], [23], authors propose models on Internet pricing, but they focus on customer pricing strategy and issues to provide differentiated service. Our work focused on the "interactions" between the higher tier and lower tier ISPs. In [21], authors consider a network of local and transit ISPs and show that positive profit is achieved using threat strategies. Our work provide a methodology to obtain the optimal pricing for higher tier ISP charging the local ISPs.

In [1], authors provide a novel model and the work is a pioneering work of studying ISP's interaction. The model is for two-layers and shows the price for the network to maximize its revenue. The work also shows the revenue per unit bandwidth increases and overall performance of each user improves when the number of users increases. In [12], authors investigate the issues of revenue maximization and network scalability of ISP, where there is only one higher tier ISP. The work also show the rationale for an ISP to perform network upgrade. Our model can be regarded as a generalization to the above models in which multiple higher tier ISPs present to allow competition, and peering links are allowed for geographically close ISPs to provide alternative transit service.

\section{Conclusion}

In this work, we investigate in the interactions between two layers of ISPs. The lower tier ISPs can transmit traffic to other lower tier ISPs and they want to obtain the maximum utilities while minimizing the congestion cost and payment. The higher tier ISPs, want to attract more customers (or lower tier 
ISPs) and maximize their revenue or profit. However, they cannot price the service at will since there is competition among the higher tier ISPs. The higher tier ISPs also need to determine the appropriate amount of bandwidth allocation to their customers so as to avoid monopoly by few customers. In this paper, we show how the lower tier ISPs can determine the appropriate traffic transmission and routing so as to maximize their utility. We also present a distributed algorithm for higher tier ISPs to allocate bandwidth resource such that resource monopoly can be avoided. We show the methodology for higher tier ISPs to compute their optimal prices where their maximum revenues are reached. Finally, we demonstrate through experiments that a higher tier ISP can obtain a larger revenue in all circumstances if it performs network upgrade, especially when we scale up the network. The above models are particularly interesting since one can use them to understand the economic impact as well as the behavior as the Internet grows.

Though this work provides some answers for the current Internet, there is room for future work. In our work, the higher tier ISP issues a single price and provides the same quality of service to all lower tier ISPs. To accommodate the situation that lower tier ISPs having different demands in the quality of transit service, higher tier ISPs should provide differentiated service according to the different demands. One way to provide differentiated service is to have multiple service classes, where customers in higher class have premium service than the customers in lower class. Besides allocating resource to different customers, higher tier ISPs will need to find an efficient algorithm to allocate its resource to different classes, as well as finding the optimal price in each class.

Moreover, the Internet is consisting of more than two tiers of ISPs. A network model which can accommodate more tiers of ISPs is a better and a more accurate representation of the Internet. However, the model and the interaction between the ISPs becomes much more complicated. For example, the change in unit price of tier 1 ISP may be extended to tier 3 ISPs. Our work provides a basic investigation for the above complicated problems. ISP can apply our model to study various issues and narrow down the design space.

\section{REFERENCES}

[1] T. Basar and R. Srikant. Revenue-maximizing pricing and capacity expansion in a many-user regime. In IEEE INFOCOM, pages 1556-1563, New York, June 2002.

[2] D. P. Bertsekas and J. N. Tsitsiklis. Parallel and Distributed Computation. Prentice Hall, 1989.

[3] G. Cardano. Cubic formula from the Mathworld.

[4] N. Christin and J. Chuang. On the cost of participating in a peer-to-peer network. In Proceedings of the Third International Workshop on Peer-to-Peer Systems (IPTPS'04), volume 3279, pages 22-32, San Diego, CA., February 2004.

[5] L. He and J. Walrand. Pricing and revenue sharing strategies for internet service providers. In IEEE INFOCOM, pages 942-951, Miami, March 2005.

[6] L. He and J. Walrand. Pricing differentiated internet services. In IEEE INFOCOM, pages 195-204, Miami, March 2005. 
[7] G. Huston. Interconnection, peering and settlements., 1998.

[8] J. W. L. Jiang, D.-M. Chiu, and J. C. S. Lui. On the interaction of multiple overlay routing. In Journal of Performance Evalaution, volume 62(1-4), pages 229-246, October 2005.

[9] F. Kelly. Charging and rate control for elastic traffic. In European Trans. on Telecommunications, volume 8, pages 33-37, 1997.

[10] J.-J. Laffont, S. Marcus, and P. R. J. Tirole. Internet interconnection and the off-net cost pricing principle. In Institut d'economic Industrielle (IEDI), Mimeo, France, April 2001.

[11] R. Lam, D.-M. Chiu, and J. C. S. Lui. On the access pricing and network scaling issues of wireless mesh networks. In IEEE Transactions on Computers, volume 56(1), pages 1456-1469, November 2007.

[12] S. C. M. Lee, J. W. J. Jiang, D.-M. Chiu, and J. C. S. Lui. Interaction of isps: Distributed resource allocation and revenue maximization. In Transaction of Parallel and Distributed System, volume 19(2), pages 204-218, Feburary 2008.

[13] T. Ma, D.-M. Chiu, and J. C. S. Lui. Incentive and service differentiation in p2p networks: A game theoretic approach. In IEEE/ACM Transactions on Networking, volume 14(5), pages 978-991, October 2006.

[14] P. Marbach. Pricing differentiated services networks: Bursty traffic. In IEEE INFOCOM, pages 650-658, Alaska, March 2001.

[15] P. Marbach. Priority service and max-min fairness. In IEEE INFOCOM, volume 11(5), pages 733-746, New York, March 2002.

[16] W. B. Norton. A business case for isp peering. In NANOG, Available at http://www.equinix.com/pdf/whitepapers/Business_case.pdf.

[17] A. M. Odlyzko. The economics of the internet: Utility, utilization, pricing, and quality of service. In AT\&T Research Lab, Tech Report, September 1998.

[18] A. M. Odlyzko. Pricing and architecture of the internet: Historical perspectives from telecommunications and transportation. In The 32nd Research Conference on Communication, Information and Internet Policy, March 2004.

[19] M. J. Osborne and A. Rubinstein. A Course in Game Theory. The MIT Press, 1957.

[20] S. S. Rao. Engineering Optimization: Theory and Practice Third Edition. A Wiley-Interscience Publication, 1996.

[21] S. Shakkottai and R. Srikant. Economics of network pricing with multiple isps. In IEEE INFOCOM, volume 1, pages 184-194, Miami, March 2005.

[22] S. Shenker, D. Clark, D. Estrin, and S. Herzog. Pricing in computer networks: Reshaping the research agenda. In ACM SIGCOMM Computer Communication Review, volume 26, pages 19-43, New York, USA, 1996.

[23] J. Shu and P. Varaiya. Pricing network services. In IEEE Infocom 2003, volume 2, pages 1221-1230, April 2003.

[24] J. W. Stewart. BGP4 : Inter-Domain Routing in the Internet. Addison Wesley, 1999.

[25] F. Wang and L. Gao. On inferring and characterizing internet routing policies. In Proceedings of the 3rd ACM SIGCOMM conference on Internet measurement, pages 15-26, October 2003.

[26] H. Wang, D.-M. Chiu, and J. C. S. Lui. Inter-as inbound traffic engineering via aspp. In IEEE Transactions on Network and Service Management, volume 4(1), pages 62-70, June 2007. 